\title{
Construction and validation of an RNA-binding protein- associated prognostic model for colorectal cancer
}

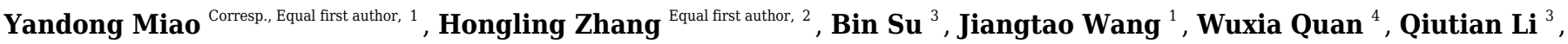 \\ Denghai Mi ${ }^{\text {Corresp. } 1,5}$ \\ ${ }^{1}$ The First Clinical Medical College, Lanzhou University, Lanzhou, Gansu, China \\ 2 Cancer Ward, Palliative Medical Center, New Kunhua Hospital, kunming, Yunnan, China \\ 3 Department of Oncology, The 920th Hospital of the Chinese People's Liberation Army Joint Logistic Support Force, kunming, Yunnan, China \\ 4 Qingyang People's Hospital, Qingyang, Gansu, China \\ 5 Gansu Academy of Traditional Chinese Medicine, Lanzhou, China \\ Corresponding Authors: Yandong Miao, Denghai Mi \\ Email address: miaoyd19@Izu.edu.cn, mi.dh@outlook.com
}

Colorectal cancer $(C R C)$ is one of the most prevalent and fatal malignancies, and novel biomarkers for the diagnosis and prognosis of CRC must be identified. RNA-binding proteins (RBPs) are essential modulators of transcription and translation. They are frequently dysregulated in various cancers and are related to tumorigenesis and development. The mechanisms by which RBPs regulate CRC progression are poorly understood and no clinical prognostic model using RBPs has been reported in CRC. We sought to identify the hub prognosis-related RBPs and to construct a prognostic model for clinical use. mRNA sequencing and clinical data for CRC were obtained from The Cancer Genome Atlas database (TCGA). Gene expression profiles were analyzed to identify differentially expressed RBPs using R and Perl software. Hub RBPs were filtered out using univariate Cox and multivariate Cox regression analysis. We used functional enrichment analysis, including Gene Ontology and Gene Set Enrichment Analysis, to perform the function and mechanisms of the identified RBPs. The nomogram predicted overall survival (OS). Calibration curves were used to evaluate the consistency between the predicted and actual survival rate, the consistency index (c-index) was calculated, and the prognostic effect of the model was evaluated. Finally, we identified 178 differently expressed RBPs, including 121 up-regulated and 57 down-regulated proteins. Our prognostic model was based on nine RBPs (PNLDC1, RRS1, HEXIM1, PPARGC1A, PPARGC1B, BRCA1, CELF4, AEN, and NOVA1). Survival analysis showed that patients in the high-risk subgroup had a worse OS than those in the low-risk subgroup. The area under the curve value of the receiver operating characteristic curve of the prognostic model is 0.712 in the TCGA cohort and 0.638 in the GEO cohort. These results show that the model has a moderate diagnostic ability. The c-index of the nomogram is 0.77 in the TCGA cohort and 0.73 in the GEO 
cohort. We showed that the risk score is an independent prognostic biomarker and that some RBPs may be potential biomarkers for the diagnosis and prognosis of CRC. 


\section{Construction and validation of an RNA-binding protein-}

\section{2 associated prognostic model for colorectal cancer}

3

4 Yandong Miao ${ }^{1 * \dagger}$, Hongling Zhang ${ }^{2 *}$, Bin $\mathrm{Su}^{3}$, Jiangtao Wang ${ }^{1}$, Wuxia Quan ${ }^{4}$, Qiutian $\mathrm{Li}^{3}$,

5 Denghai Mi $1,5 \dagger$

6

$7 \quad{ }^{1}$ The First Clinical Medical College of Lanzhou University, Lanzhou City, Gansu Province, PR

8 China

$9 \quad{ }^{2}$ Cancer Ward, Palliative Medical Center, New Kunhua Hospital, Kunming City, Yunnan

10 Province, PR China

$11{ }^{3}$ Department of Oncology, The 920th Hospital of the Chinese People's Liberation Army Joint

12 Logistic Support Force, Kunming City, Yunnan Province, PR China

$13{ }^{4}$ Qingyang People's Hospital, Qingyang City, Gansu Province, PR China

$14{ }^{5}$ Gansu Academy of Traditional Chinese Medicine, Lanzhou City, Gansu Province, PR China

$15{ }^{*}$ These authors have equal contributions.

$16 \dagger$ Corresponding Author:

17 1. Denghai Mi: mi.dh@outlook.com;

18 2. Yandong Miao: miaoyd19@1zu.edu.cn. 
33 Abstract

34 Colorectal cancer (CRC) is one of the most prevalent and fatal malignancies, and novel

35

36

37

38

39

40

41

42

43

44

45

46

47

48

49

50

51

52

53

54

55

56

57

58

59

60

61

62

63

64

biomarkers for the diagnosis and prognosis of CRC must be identified. RNA-binding proteins (RBPs) are essential modulators of transcription and translation. They are frequently dysregulated in various cancers and are related to tumorigenesis and development. The mechanisms by which RBPs regulate CRC progression are poorly understood, and no clinical prognostic model using RBPs has been reported in CRC. We sought to identify the hub prognosis-related RBPs and to construct a prognostic model for clinical use. mRNA sequencing and clinical data for CRC were obtained from The Cancer Genome Atlas database (TCGA). Gene expression profiles were analyzed to identify differentially expressed RBPs using R and Perl software. Hub RBPs were filtered out using univariate Cox and multivariate Cox regression analysis. We used functional enrichment analysis, including Gene Ontology and Gene Set Enrichment Analysis, to perform the function and mechanisms of the identified RBPs. The nomogram predicted overall survival (OS). Calibration curves were used to evaluate the consistency between the predicted and actual survival rate, the consistency index (c-index) was calculated, and the prognostic effect of the model was assessed. Finally, we identified 178 differently expressed RBPs, including 121 up-regulated and 57 down-regulated proteins. Our prognostic model was based on nine RBPs (PNLDC1, RRS1, HEXIM1, PPARGC1A, PPARGC1B, BRCA1, CELF4, AEN, and NOVA1). Survival analysis showed that patients in the high-risk subgroup had a worse OS than those in the low-risk subgroup. The area under the curve value of the receiver operating characteristic curve of the prognostic model is 0.712 in the TCGA cohort and 0.638 in the GEO cohort. These results show that the model has a moderate diagnostic ability. The c-index of the nomogram is 0.77 in the TCGA cohort and 0.73 in the GEO cohort. We showed that the risk score is an independent prognostic biomarker and that some RBPs may be potential biomarkers for the diagnosis and prognosis of CRC.

\section{Introduction}

Colorectal cancer (CRC) is the fourth most lethal cancer, with about 900,000 deaths annually. It is the second most common cancer in women and the third most common cancer in men (Dekker et al., 2019). CRC has a complicated biological progression affected by multiple factors, such as lifestyle, obesity, and environmental factors. Its development involves profound changes at various molecular levels, including in the transcriptome, genome, epigenome, and proteome 
65 (Murphy et al., 2018). Despite significant advances in diagnostic and therapeutic approaches, the

66

67

68

69

70

71

72

73

74

75

76

77

78

79

80

81

82

83

84

85

86

87

88

89

90

91

92

93

94

95

96

97

average 5-year survival rate of CRC is 48\% (Sharma, 2020); the 5-year survival rate for patients with metastatic CRC is only 10\% (Lafitte et al., 2020). CRC diagnosis typically relies on imaging evaluation, molecular cancer biomarkers, and histopathological examination. It is difficult to obtain an early diagnosis for CRC (Jung et al., 2020; Luo et al., 2019), leading to a high mortality rate for CRC patients. Consequently, we need to understand the molecular mechanisms of CRC better and explore more efficient early screening and diagnosis methods to improve the treatments and quality of life for CRC patients.

RNA-binding proteins (RBPs) are generally considered to be proteins that bind RNA through one or more globular RNA-binding domains and alter the fate or function of the bound RNA (Hentze et al., 2018). RBPs are crucial to RNA metabolism and regulate the spatial, temporal, and functional dynamics of RNA. Altered RBP expression affects cell physiology and the RNA processes from pre-mRNA splicing to protein translation. Current genetic and proteomic data and results from animal models suggest that RBPs are precipitated in many human diseases, from neurological disorders to cancer (Lukong et al., 2008). Approximately 1,542 RBP genes have been identified through whole-genome screening of the human genome (Gerstberger et al., 2014). These RBPs contribute to genetic regulation over a wide range of cellular and developmental processes (Kong \& Lasko, 2012). Previous research has demonstrated that RBPs, including the human pumilio proteins, PUM1, and PUM2, affect human disease genes related to cancer and neurological and cardiovascular diseases (Bohn et al., 2018).

Previous studies have shown that RBPs are critical modulators of transcription and translation, which are often dysregulated in cancer and are associated with cancer transformation, tumorigenesis, and poor prognosis (Dang et al., 2017; Kim et al., 2015; Wang et al., 2019a). Recent research has shown that RBPs regulate the dynamic balance of proliferation, migration, differentiation, and senescence of intestinal epithelial cells, interacting with crucial pathways in CRC (Chatterji \& Rustgi 2018). A limited number of RBPs have been well-studied and have been found to play vital roles in regulating the initiation and progression of cancer (van Kouwenhove et al., 2011). For example, CRD-BP is necessary to induce both beta TCP1 and cMyc through beta-catenin signaling in CRC cells (Noubissi et al., 2006). Heterogeneous ribonucleoprotein A0 promotes excessive mitosis and the growth of CRC cells (Konishi et al., 2020). Two RBPs have been explored that had obvious effects on the metastasis and overall 
98 99

100

101

102

103

104

105

106

107

108

109

110

111

112

113

114

115

116

117

118

119

120

121

122

123

124

125

126

127

128

129

survival (OS) of CRC patients (Zhou, B et al.,2018). However, RBPs regulate CRC progression through poorly understood mechanisms. Previous studies have shown that twelve identified RBPs may be promising predictors of $\mathrm{CRC}$ but no further verification at the protein expression level (Fan, Xuehui, et al.,2020). Zhang, Zhen et al. was constructed a prognostic risk score model based on four RBPs and explore their prognosis value for CRC patients. But no further analysis of the potential prognosis proteins co-expressed with RBPs (Zhang, Zhen, et al.,2020). A comprehensive functional study of RBPs is needed to better understand their role in CRC. In the current study, mRNA sequencing and clinical data of CRC were obtained from The Cancer Genome Atlas (TCGA) database. We illuminated abnormally expressed RBPs from normal and cancer samples through a series of bioinformatics analyses and comprehensively identified their potential functions and molecular mechanisms. Besides, we also identified several potential prognosis proteins co-expressed with RBPs. Our study identified some CRC-related RBPs that assist in understanding the molecular mechanisms of CRC progression. We developed a novel risk profile as an independent prognostic biomarker for risk stratification in CRC patients. Our research design and analysis flow chart are shown in Figure 1.

\section{Materials \& Methods}

\section{Data sources}

mRNA sequencing and clinical data for CRC were downloaded from the TCGA database (Data Release 24.0-May 7, 2020, https://portal.gdc.cancer.gov/). Detailed data selection criteria described in our previous study: filter criteria of mRNA sequencing: (1) the keywords of cases are [Primary Site] "colon, rectosigmoid junction, and rectum," [Program] "TCGA," [Project] “TCGA-COAD, TCGA-READ,", [Disease Type] "Adenomas and Adenocarcinomas."(2) The keywords of files are [Data Category] "Transcriptome Profiling," [Data Category] "Gene Expression Quantification," [Experimental Strategy] "RNA-Seq,"” [Workflow Type] "HTSeq FPKM"), filter criteria of clinical data: [Data Category] "Clinical," [Data Format] "BCR XML”). (Miao et al. 2020). We obtained 692 mRNA expression profiles of CRC. Among them, 51 (7.4\%) samples were normal and 641 samples (92.6\%) were cancerous. The TCGA-CRC cohort consisted of 624 patients. The clinicopathological features of the patients are listed in Table S1. The GEO dataset was chosen mainly as a validation dataset to prove the accuracy of analysis results based on TCGA dataset. The data access (GSE39582, GSE87211) was obtained from the Gene Expression Omnibus (GEO, https:/www.ncbi.nlm.nih.gov/geo/) for verification 
130

131

132

133

134

135

136

137

138

139

140

141

142

143

144

145

146

147

148

149

150

151

152

153

154

155

156

157

158

159

160

161

162

studies. The GSE39582 dataset consisted of 585 samples

(https://www.ncbi.nlm.nih.gov/geo/query/acc.cgi?acc=GSE39582), including 19 colon mucosa samples and 566 stage I-IV colon adenocarcinoma samples. The GSE87211 dataset included 363 samples (https:/www.ncbi.nlm.nih.gov/geo/query/acc.cgi?acc=GSE87211), including 160 rectal mucosa samples and 203 rectal tumor samples (Hu et al., 2018; Marisa et al., 2013). We merged the two datasets through a Perl script. Raw data from gene chips were normalized using the RMA algorithm provided by the R-package "limma" (Ritchie et al., 2015). Perl script and the Rpackage "sva" were used to merge microarray data and decrease heterogeneity between the two studies (Zhang et al., 2019).

\section{Identification of differential expression RBPs.}

RNA binding proteins (RBPs) were obtained from the literature (Gerstberger et al., 2014). Perl software was used to extract and integrate the data. We used the Wilcox Test in R-package "limma" to screen differentially expressed RBPs. False-positive discovery (FDR) $<0.05$ and Log2 | (fold change, FC) |>1 was set as the cutoffs. R-package "pheatmap" was used to analyze Bidirectional hierarchical clustering and draw a heat map.

\section{Functional enrichment analysis of RBPs}

Functional annotation and enrichment analysis of up-regulated and down-regulated RBPs were based on the R-packages "clusterProfiler", “org.Hs.eg.db", "ggplot2", and "enrichplot", which classified Gene Ontology (GO) to the Biological Processes (BP), Cellular Components (CC), and Molecular Functions (MF). FDR $<0.05$ was set as the cutoff. Kyoto Encyclopedia of Genes and Genomes (KEGG) pathways analysis was performed by Gene Set Enrichment Analysis (GSEA). It was considered statistically significant that the number of permutations was set to $1,000, P$-value $<0.05$, and FDR $<0.25$.

\section{Protein-protein interaction network constructed and module selection}

The STRING database (http://www.string-db.org/) (von Mering et al., 2003) was used to evaluate the protein-protein interaction (PPI) information from differently expressed RBPs. Cytoscape software (version 3.7.2) was used to construct and visualize the PPI network. The essential genes and modules were selected in the PPI network using the Molecular Complex Detection (MCODE) plug-in with MCODE and node count numbers more significant than five (Bader \& Hogue, 2003).

\section{Construction prognosis model of RBPs and survival analysis}


163 Univariate Cox regression analysis was used to screen out the RBPs that had a significant

164

165

166

167

168

169

170

171

172

173

174

175

176

177

178

179

180

181

182

183

184

185

186

187

188

189

190

191

192

193

relationship with the OS of CRC patients $(\mathrm{p}<0.05)$. We used multivariate Cox regression analysis to construct an optimal model of RBPs. We used Kaplan-Meier analysis and the log-rank test $(p<0.05)$ to assess survival. Each patient's risk score was calculated using the following formula: Risk score $=\sum_{n=1}^{n}$ Coefficient $(n) *$ gene expression $(n)$, Coefficient $(n)$ representing the regression coefficient and gene expression $(n)$ indicating the relative expression levels of each prognosis-associated RBPs normalized by z-score in the prognostic risk score model, respectively (Liu et al., 2019, Zhang, Zhen, et al.,2020). Survival information was obtained from the clinical data of the aforementioned samples. The median risk score was chosen as the cutoff value for the CRC cohort dichotomy, and the CRC patients were categorized into high and low-risk groups. We used the R-packages "survminer" and "survival" to draw the survival curve according to the high and low-risk values. The Receiver Operating Characteristic (ROC) curve was used to examine the specificity and sensitivity of survival prediction by the protein characteristic risk score and was drawn using the R-package "survival ROC". The area under curve (AUC) value is an indicator of prognostic accuracy (Sachs, 2017). We drew a heat map, risk curve, and survival state diagram based on the different risk scores of our patients. Independent prognostic proteins were identified using univariate and multivariate Cox hazard regression analysis. Seven hundred and fifty-eight CRC patient samples with prognostic information from the GSE39582 and GSE87211 datasets were used as verification cohorts to verify the prognostic model's predictive ability. Protein coexpression was analyzed using the R-packages "ggalluvial”, "ggplot2", and "dplyr".

Immunohistochemistry (IHC) analysis

We used the Human Protein Atlas (HPA, http://www.proteinatlas.org/) database to explore the expression level of the hub RBPs in the normal and tumor samples. Analysis of each protein and its corresponding cancer type in patients, using IHC analysis based on tissue microarrays (TMAs), is presented for a majority of the protein-coding genes. IHC staining was conducted according to prior published literature: the staining index scores were distributed as follows: staining intensity (negative:0; Weak:1; Moderate: 2; Strong:3), positive staining (0: $<5 \% ; 1: 5-25 \% ; 2: 26-50 \% ; 3$ : 51-75\%;4: >75\%). The staining index score was computed through multiplying the staining intensity score by the positive staining score, which ranged from 0 to 12 (Zhang, Zhen, et al.,2020).

\section{Construction the nomogram}

Peer] reviewing PDF | (2020:11:55733:1:1:NEW 25 Feb 2021) 
194 We used the expression level of RBPs by the R-package "rms" to construct the nomogram and 195 predict the likelihood of OS. Calibration curves were used to estimate the consistency between 196 predicted and actual survival, and the performance of the model in predicting prognosis was 197 evaluated by the C-index. C-index values of 0.5 and 1.0 represent a random probability and an 198 excellent ability to predict survival, respectively.

199

\section{Results}

201 Identification of differently expressed RBPs in CRC patients

202 One thousand, five hundred and forty-two RBPs were used in our study. One hundred and

203 seventy-eight differently expressed RBPs were excluded, and 121 up-regulated, and 57 down-

204 regulated RBPs were included in our study (Figure 2).

205 Identification of involved signaling pathways of the differently expressed RBPs We divided the selected RBPs into up-or down-regulated expression groups to explore their function and mechanisms. Functional enrichment analysis was conducted using R software. The results showed that $105 \mathrm{GO}$ terms of $\mathrm{BP}, 21 \mathrm{GO}$ terms of $\mathrm{CC}$, and $48 \mathrm{GO}$ terms of MF were significant in the up-regulated group (FDR $<0.05$ ). Seventy-three GO terms of BP, 3 GO terms of $\mathrm{CC}$, and $25 \mathrm{GO}$ terms of MF were significant in the down-regulated group (FDR $<0.05$ ). The upregulated RBPs were mainly enriched in ncRNA metabolic process during BP analysis, ncRNA processing, nucleic acid phosphodiester bond hydrolysis, RNA phosphodiester bond hydrolysis, and ribonucleoprotein complex biogenesis (Figure 3A). The down-regulated differently expressed RBPs were significantly enriched in mRNA processing, RNA splicing, defense response to a virus, response to a virus, and translation regulation (Figure 3B). During the $\mathrm{CC}$ analysis, the upregulated RBPs were enriched in the nucleolar part, cytoplasmic ribonucleoprotein granule, 217 ribonucleoprotein granule, nuclear envelope, and chromatin (Figure 3A). In contrast, the down218 regulated RBPs were enriched in the endolysosome membrane, apical dendrite, and endolysosome 219 (Figure 3B). In terms of MF, the up-regulated differently expressed RBPs were notably enriched during catalytic activity, when acting on RNA, and during nuclease activity, ribonuclease activity, endonuclease activity, and tRNA binding (Figure 3A). The down-regulated differently expressed RBPs were significantly enriched in mRNA binding, AU-rich element-binding, mRNA 3'-UTR AU-rich region binding, mRNA 3'-UTR binding double-stranded RNA binding (Figure 3B). The top-10 terms of BP, CC, and MF are shown in Figure 3. The detailed information of GO the terms 
226

227

228

229

230

231

232

233

234

235

236

237

238

239

240

241

242

243

244

245

246

247

248

249

250

251

252

253

254

255

256

257

258

RBPs showed that 66/165 gene sets were up-regulated in phenotype h; 35 gene sets were significant at FDR $<25 \%$ and 99/165 gene sets were up-regulated in phenotype 1 , and 55 gene sets were significant at FDR $<25 \%$. The top-five up-regulated gene sets were basal cell carcinoma (NES=2.15, $\mathrm{P}=0.000$ ), dilated cardiomyopathy ( $\mathrm{NES}=2.11, \mathrm{P}=0.000$ ), glycosaminoglycan biosynthesis chondroitin sulfate $(\mathrm{NES}=2.08, \mathrm{P}=0.000$ ), complement and coagulation cascades (NES=2.06, $\mathrm{P}=0.002$ ), and hypertrophic cardiomyopathy hcm (NES=1.91, $\mathrm{P}=0.002)$, while the top-five downregulated gene sets were RNA degradation ( $\mathrm{NES}=-2.08, \mathrm{P}=0.008$ ), valine leucine and isoleucine degradation $(\mathrm{NES}=-2.06, \mathrm{P}=0.004)$, ubiquitin-mediated proteolysis $(\mathrm{NES}=-2.02$, $\mathrm{P}=0.006)$, propanoate metabolism $(\mathrm{NES}=-1.95, \mathrm{P}=0.006$ ), and oocyte meiosis $(\mathrm{NES}=-1.92$, $\mathrm{P}=0.014$ ) (Figure 3C-3L). The detailed GSEA analysis is shown in Table S3.

\section{PPI network construction and hub modules screening}

We constructed a PPI network with 147 nodes and 543 edges based on the STRING database's data using Cytoscape software to further explore the roles of differentially expressed RBP in CRC (Figure 4A). The co-expression network was constructed by MODE Plug-in to the study potential hub modules and the top-four crucial modules. The compulsory modules 1, 2, 3, and 4 consisted of 18 nodes and 144 edges; 9 nodes and 28 edges; 5 nodes and ten edges; and 6 nodes and 12 edges, respectively (Figure 4B). RBPs in the compulsory modules 1-4 were mainly enriched in ribosome biogenesis, ncRNA processing, mRNA processing, mRNA binding, defense response to a virus, response to a virus, DNA alkylation, and DNA methylation. The PPI network is shown in Table S4.

\section{Construction prognosis model of RBPs and survival analysis}

One hundred forty-seven differently expressed RBPs were identified from the PPI network. To further exploring the prognostic value of these RBPs, we carried out a univariate Cox regression analysis and screened out 15 potential prognostic-associated key RBPs, including six Low-Risk BAPs $(H R<1, p<0.05)$ and nine High-Risk RBPs $(H R>1, p<0.05$, Figure $5 A)$. Nine RBPs were filtered out by multivariate COX regression analysis (Figure 5B). The prognosis model was constructed based on these nine RBPs. The full name and coefficients of these proteins are shown in Table 1.

The risk score for each patient was calculated based on the following formula:

Risk score $=0.2943 \times$ expression level of PNLDC1 $+0.4835 \times$ expression level of RRS $1+0.3609$ $\times$ expression level of HEXIM1 $+(-0.1757) \times$ expression level of PPARGC1A $+(-0.3660) \times$ expression of PPARGC1B $+(-0.4157) \times$ expression level of BRCA1 $+0.5460 \times$ expression level of CELF4 $+0.4375 \times$ expression level of AEN $+0.1639 \times$ expression level of NOVA1. 
259 The risk score was applied to predict prognosis, and $586 \mathrm{CRC}$ patients were divided into high-risk 260 and low-risk subgroups according to the median risk score. Kaplan-Meier cumulative curves 261 showed a significant difference in OS between the high-risk and low-risk groups. Patients with 262 high-risk scores had a worse OS than those with low-risk scores (Figure 5C). A ROC analysis was 263 used further to assess the prognostic accuracy of the prognosis model. Our results indicated that 264 the AUC value was 0.712 (Figure 5D), which showed a moderate diagnostic ability. An elevated 265 risk score was correlated with a decreased survival time and an increase in mortality (Figure 5E). 266 Age, clinical-stage, primary tumor size (T), regional lymph node involvement (N), distant 267 metastasis (M), and risk score were significantly associated with OS in the univariate independent 268 prognostic analysis $(\mathrm{P}<0.001$, Figure 5F, Table 2$)$. The multivariate independent predictive 269 analysis demonstrated that age, T, and risk scores were also independent prognostic predictors $270(\mathrm{P}<0.05$, Figure 5G, Table 2). We assessed whether the nine-RBPs predicting model had an 271 equivalent prognostic value in other CRC cohorts and used the same formula in the validation 272 group in which data come from the GSE39582 and GSE87211 datasets. The result showed that 273 patients with high-risk scores also had a worse OS than those with low-risk scores; the risk score 274 was also an independent prognostic predictor in the GSE39582 and GSE87211 cohorts (Figure 6, 275 Table 3). These results showed that the prognostic model, based on the nine RBPs, had higher 276 sensitivity and specificity.

\section{Construction of a nomogram based on the nine crucial RBPs}

278 A nomogram can quantitatively identify an individual's clinical risk by combining multiple risk 279 factors (Liang et al., 2015; Won et al., 2015). We used the nomogram to predict the probability of 280 1-, 3- and 5-year OS by merging the nine RBPs signatures for TCGA-CRC and verified these 281 results using the GEO data sets (Figure 7A). The distribution point of each RBPs was proportional 282 to its risk contribution to the survival and was normalized to the distribution of 0 to 100 . We 283 calculated each patient's total points by summarizing the number of points for all RBPs and 284 estimated the survival rates by drawing a vertical line between each prognosis axis and the total 285 point axis. This tool may help practitioners make clinical decisions for CRC patients. The 286 calibration curve demonstrated that the actual survival rate matched the predicted 1-, 3- and 5287 year survival rate. The C-index was 0.77 in the TCGA database (Figure 7B). The nomogram was 288 verified in the GEO cohorts, the C-index was 0.73, and the 1-, 3- and 5- year calibration curves 289 are shown in Figure 7C.

\section{Co-expression analysis of RBPs}


291 To explore more potential prognosis proteins, we used the nine RBPs in the model for co-

292 expression analysis. CELF4 and LUZP4 were co-expressed; NOVA1 was co-expressed with 293 RBFOX3, ELAVL4, DZIP1, SAMD4A, ZCCHC24, AFF3, RBM20, ENOX1, RBPMS2, and 294 CPEB1. PPARGC1A was co-expressed with RBM47, RAVER2, and CPEB3. PPARGC1B was 295 co-expressed with RBM47, RAVER2, and LRRFIP2. RRS1 was co-expressed with BOP1

296 ( $\mid$ correlation coefficient $\mid>0.6, \mathrm{P}<0.001$, Figure 8). The co-expression analysis is shown in 297 Table S5.

\section{Validation of the expression of hub RBPs}

299 The immunohistochemistry results of the HPA database were used to explore the expression of 300 the nine hubs RBPs in CRC, and the result showed that RRS1 and BRCA1 were highly 301 expressed in the CRC tissues compared with normal tissues. However, PNLDC1 was more 302 highly expressed in the normal colorectal tissues than in the cancer tissues. HEXIM1 was highly 303 expressed in both normal and colorectal tissues. In contrast, CELF4 was not expressed in normal 304 and colorectal tissues. Furthermore, the protein expression of NOVA1 was not significantly 305 different between normal and CRC tissues (Figure 9).

\section{Discussion}

307 RNA regulatory-mediated RBPs are involved in maintaining homeostasis and in cancer progression. However, the tumor-related functions of most RBPs and the detailed mechanisms of their anti-tumor effects remain to be explored (Jonas et al., 2020; Konishi et al., 2020). We screened out 178 differently expressed RBPs in normal and tumor tissues from the TCGA-CRC data set. We then comprehensively analyzed the appropriate biological processes and pathways and constructed a PPI network and co-expression network of these RBPs. We applied univariate and multivariate COX regression analysis, survival analysis, and ROC analyses and constructed a nomogram to predict prognoses and further explore the ten RBPs related to the clinicopathological characteristics of CRC patients. We constructed a risk model based on nine prognostic-associated core RBP genes to predict the prognosis of CRC. These discoveries may provide novel biomarkers to use in the diagnosis and prognosis of CRC patients.

318 The results of our functional pathway enrichment analysis showed that differently expressed RBPs 319 were abundantly enriched in the ncRNA metabolic process, ncRNA processing, ribonucleoprotein 320 complex biogenesis, mRNA processing, regulation of translation, nucleolar part, endolysosome membrane, apical dendrite, catalytic activity, RNA activity, tRNA binding, mRNA binding, AUrich element binding, and mRNA 3'-UTR AU-rich region binding. Previous studies demonstrated 
323 that RBPs regulate various aspects of RNA metabolism and tumorigenesis (Mitobe et al., 2020; 324 Zhu et al., 2020). The ncRNA regulates proliferation checkpoints and inflammatory gene 325 expression in CRC (Ma et al., 2019). The ribonucleoprotein complex can promote excessive 326 mitosis and the growth of colorectal cancer cells (Konishi et al., 2020). mRNA processing, tRNA 327 binding, and mRNA binding play an essential role in CRC growth and metastasis (Kong \& Wang, 328 2020; Wang et al., 2019b). KEGG pathways analysis showed that the aberrantly expressed RBPs 329 also regulated basal cell carcinoma, dilated cardiomyopathy, glycosaminoglycan biosynthesis, 330 chondroitin sulfate, complement and coagulation cascades, and hypertrophic cardiomyopathy.

331

332 We constructed a PPI network based on differently expressed RBPs and obtained four key 333 modules, including 38 core RBPs. Many of these essential RBPs have been shown to play a crucial 334 role in the development and progression of cancers. BOP1 is a direct transcription target of $\beta$ 335 catenin/TCF4, which induced cell migration and experimental metastasis of CRC cells (Qi et al., 336 2016). DKC1 may regulate CRC angiogenesis and metastasis by directly activating HIF-1 $\alpha$ 337 transcription (Hou et al., 2020). Ji et al. (2019) showed that PTBP2 could elevate translational 338 levels of RUNX2, which plays a vital role in CRC metastasis. NOVA1 expression controls the 339 proliferation and invasive properties of CRC cells by enhancing IL-6/JAK2/STAT3 signal 340 transduction, and in turn, up-regulating matrix metalloproteinases (MMPs) 2, 7, and 9 (Hong et 341 al., 2019). CRC associated with inflammation by activation initiated TLR3 and TLR7 (He et al., 342 2017). EZH2 expression and activity are associated with colorectal carcinogenesis and are most 343 often expressed in highly intraepithelial lesions (Bremer et al., 2019). The PPI network's module 344 analysis showed that $\mathrm{CRC}$ is related to ribosome biogenesis, ncRNA processing, ribonucleoprotein 345 complex biogenesis, ncRNA metabolic process, mRNA processing, mRNA binding, and RNA 346 splicing.

347

348 Nine RBPs, including PNLDC1, RRS1, HEXIM1, PPARGC1A, PPARGC1B, BRCA1, CELF4, 349 AEN, and NOVA1, were identified as prognosis-associated core RBPs based on multivariate Cox 350 regression analysis. Previous studies reported that the expression of RRS1 may promote the 351 development of colon cancer (Wu et al., 2017) and that HEXIM1 is a positive regulator of p53 352 (Lew et al., 2012). PPARGC1A and PPARGC1B have also been demonstrated to contribute to 353 CRC susceptibility (Lin et al., 2019). Studies have also shown that BRCA1 mutation carriers are 354 not at high risk for CRC (Cullinane et al., 2020) and that NOVA1 is expressed at higher levels in 
355 CRC cell lines (Hong et al., 2019). These RBPs are related to tumorigenesis and progression in

356

357

358

359

360

361

362

363

364

365

366

367

368

369

370

371

372

373

374

375

376

377

378

379

380

381

382

383

384

385

CRC patients, which are in-line with our results.

We constructed a risk model to predict CRC prognosis through multivariate Cox analysis based on the nine hub RBPs coding genes. The TCGA cohort was used as the training group. ROC curve analysis showed that the nine gene markers had a better diagnostic ability and could select CRC patients with poor prognosis and AUC values of ROC, which was consistent with previous reports (Jeun et al., 2019). Moreover, the validation group results (GEO cohorts) were consistent with the results from the training group. However, the molecular mechanisms of the nine RBPs contributing to CRC are still poorly understood, and further investigation into their potential mechanisms is needed.

We developed a nomogram to more accurately predict 1-, 3-, and 5-year OS. In addition to traditional clinicopathological characteristics, including tumor size, TNM stage, and tissue subtypes, gene expression can also be incorporated into predictive nomogram models to predict clinical outcomes (Reichling et al., 2019; Sjoquist et al., 2018). A nomogram for predicting 3- and 5-year recurrence-free survival in NSCLC has been reported and included a prognostic score calculated from the autophagy gene signature (Liu et al., 2019). A combination of autophagy gene markers with a prognostic element has a better prognostic value than an individual marker (Mo et al., 2019). The calibration curve showed that a nomogram could accurately predict 3- and 5-year survival probabilities (Xiong et al., 2017). We were able to show that a nomogram, including the nine RBPs signature, could better predict 1-, 3-, and 5-year survival possibility of CRC patients. These results suggest that the nine-RBPs prognostic model is of value in determining the treatment plan of CRC patients.

Co-expression analysis indicated that five RBPs were co-expressed with sixteen proteins. BOP1 is related to cell migration and experimental metastasis of CRC cells (Qi et al., 2016). The high expression of CPEB3 is related to the poor prognosis of CRC (Waku et al., 2020). ELAVL4 is upregulated in CRC patients (Huang et al., 2012). LRRFIP2 is associated with hereditary nonpolyposis CRC (Lynch Syndrome) (Morak et al., 2011). The down-regulation of RBM47 during CRC progression may promote EMT and metastasis (Rokavec et al., 2017). The effects of other proteins, including AFF3, CPEB1, DZIP1, ENOX1, LUZP4, RAVER2, RBFOX3, RBM20, 
387 388 389

390

391

392

393

394

395

396

397

398

399

400

401

402

403

404

405

406

407

408

409

410

411

412

\section{Funding}

414 No funding was received

\section{Conflicts of Interest}

416

\section{Conclusions}

\section{Acknowledgments}

\section{Author contributions} authors approved the paper.

RBPMS2, SAMD4A, and ZCCHC24, in the development of colorectal cancer is poorly understood and should be investigated further.

Our RBPs prognostic model reduces the cost of testing and is able to be applied clinically. The nine RBPs-prediction model has better survival prediction capability in CRC patients.

Nevertheless, this study has several limitations. Firstly, we designed the study based on a retrospective analysis, and prospective studies are needed to validate the future results. Secondly, the data set does not provide some clinical information, such as tumor grade in CRC, which may reduce the statistical effectiveness and dependability of multivariate Cox analysis.

We systematically investigated the prognostic value of RBPs in CRC using multiple bioinformatics analyses. These RBP may be related to the occurrence, development, invasion, and metastasis of CRC. The prognostic model of nine hubs RBPs was established, and the risk score was shown to be an independent prognostic factor for CRC. Our results will help to understand the pathogenesis of CRC and develop new therapeutic targets and prognostic biomarkers. These findings provide a comprehensive perspective for further study of the role of the hub RBPs in the pathogenesis of CRC and identify potential molecular markers for the diagnosis and treatment of CRC.

We are grateful for the availability of the data from the TCGA and GEO databases.

Conceptualization: Miao Y, Mi D; Methodology: Miao Y, Zhang H, Su B; Software: Miao Y, Zhang H, Su B; Validation: Zhang H, Su B, Li Q, Quan W; Formal analysis: Miao Y, Zhang H, Su B, Wang J; Data curation: Miao Y, Wang J, Quan W, Li Q; Writing — original draft: Miao Y, Zhang H, Bin S; Writing — review \& editing: Miao Y, Mi D; Project administration: Mi D. All

The authors declare that they have no conflicts of interest to report regarding the present study. 


\section{Data Availability}

418 The following information was supplied regarding data availability:

419 The data of clinical for this study can be found in the TCGA database 420 (https://portal.gdc.cancer.gov/). The database of validation group for this study can be found in the 421 Gene Expression Omnibus (GEO, https://www.ncbi.nlm.nih.gov/geo/) database, including 422 GSE39582, (https://www.ncbi.nlm.nih.gov/geo/query/acc.cgi?acc=GSE39582), and GSE87211 423 (https://www.ncbi.nlm.nih.gov/geo/query/acc.cgi?acc=GSE87211) datasets. The data were 424 obtained from TCGA and GEO database, strictly following the publication guidelines of TCGA 425 and GEO. Other data used and/or analyzed during the current study are available from the first or 426 corresponding author on reasonable request.

427

428

429

430

431

432

433

434

435

436

437

438

439

440

441

442

443

444

445

446

447

\section{References}

Bader GD, and Hogue CW. 2003. An automated method for finding molecular complexes in large protein interaction networks. Bmc Bioinformatics 4:2. 10.1186/1471-2105-4-2

Bohn JA, Van Etten JL, Schagat TL, Bowman BM, McEachin RC, Freddolino PL, and Goldstrohm AC. 2018. Identification of diverse target RNAs that are functionally regulated by human Pumilio proteins. Nucleic Acids Res 46:362-386. 10.1093/nar/gkx1120

Bremer SCB, Conradi LC, Mechie NC, Amanzada A, Mavropoulou E, Kitz J, Ghadimi M, Ellenrieder V, Ströbel P, Hessmann E, Gaedcke J, and Bohnenberger H. 2019. Enhancer of Zeste Homolog 2 in Colorectal Cancer Development and Progression. Digestion:1-9. 10.1159/000504093

Chatterji P, and Rustgi AK. 2018. RNA Binding Proteins in Intestinal Epithelial Biology and Colorectal Cancer. Trends Mol Med 24:490-506. 10.1016/j.molmed.2018.03.008

Cullinane CM, Creavin B, O'Connell EP, Kelly L, O'Sullivan MJ, Corrigan MA, and Redmond HP. 2020. Risk of colorectal cancer associated with BRCA1 and/or BRCA2 mutation carriers: systematic review and meta-analysis. Br J Surg. 10.1002/bjs.11603

Dang H, Takai A, Forgues M, Pomyen Y, Mou H, Xue W, Ray D, Ha KCH, Morris QD, Hughes TR, and Wang XW. 2017. Oncogenic Activation of the RNA Binding Protein NELFE and MYC Signaling in Hepatocellular Carcinoma. Cancer Cell 32:101-114.e108. 10.1016/j.ccell.2017.06.002

Dekker E, Tanis PJ, Vleugels JLA, Kasi PM, and Wallace MB. 2019. Colorectal cancer. Lancet 394:1467-1480. 10.1016/s0140-6736(19)32319-0 
448

449

450

451

452

453

454

455

456

457

458

459

460

461

462

463

464

465

466

467

468

469

470

471

472

473

474

475

476

477

478

479

480

Fan, X., Liu, L., Shi, Y., Guo, F., Wang, H., Zhao, X., Zhong, D., and Li, G. 2020. Integrated analysis of RNA-binding proteins in human colorectal cancer. World journal of surgical oncology, 18: 222. $10.1186 / \mathrm{s} 12957-020-01995-5$

Gerstberger S, Hafner M, and Tuschl T. 2014. A census of human RNA-binding proteins. Nat Rev Genet $15: 829-845.10 .1038 / \operatorname{nrg} 3813$

He L, Chen Y, Wu Y, Xu Y, Zhang Z, and Liu Z. 2017. Nucleic acid sensing pattern recognition receptors in the development of colorectal cancer and colitis. Cell Mol Life Sci 74:2395-2411. $10.1007 / \mathrm{s} 00018-017-2477-1$

Hentze MW, Castello A, Schwarzl T, and Preiss T. 2018. A brave new world of RNA-binding proteins. Nat Rev Mol Cell Biol 19:327-341. 10.1038/nrm.2017.130

Hong YG, Xu GS, Yu GY, Zhou JD, Liu QZ, Ni JS, Yan HL, Zhang W, and Hao LQ. 2019. The RNA binding protein neuro-oncological ventral antigen 1 (NOVA1) regulates IL-6 mRNA stability to enhance JAK2-STAT3 signaling in CRC. Surg Oncol 31:67-74. 10.1016/j.suronc.2019.09.009

Hou P, Shi P, Jiang T, Yin H, Chu S, Shi M, Bai J, and Song J. 2020. DKC1 enhances angiogenesis by promoting HIF-1 $\alpha$ transcription and facilitates metastasis in colorectal cancer. Br J Cancer 122:668-679. 10.1038/s41416-019-0695-z

Hu Y, Gaedcke J, Emons G, Beissbarth T, Grade M, Jo P, Yeager M, Chanock SJ, Wolff H, Camps J, Ghadimi BM, and Ried T. 2018. Colorectal cancer susceptibility loci as predictive markers of rectal cancer prognosis after surgery. Genes Chromosomes Cancer 57:140-149. $10.1002 /$ gcc. 22512

Huang MY, Wang HM, Chang HJ, Hsiao CP, Wang JY, and Lin SR. 2012. Overexpression of S100B, TM4SF4, and OLFM4 genes is correlated with liver metastasis in Taiwanese colorectal cancer patients. DNA Cell Biol 31:43-49. 10.1089/dna.2011.1264

Jeun M, Lee HJ, Park S, Do EJ, Choi J, Sung YN, Hong SM, Kim SY, Kim DH, Kang JY, Son HN, Joo J, Song EM, Hwang SW, Park SH, Yang DH, Ye BD, Byeon JS, Choe J, Yang SK, Moinova H, Markowitz SD, Lee KH, and Myung SJ. 2019. A Novel Blood-Based Colorectal Cancer Diagnostic Technology Using Electrical Detection of Colon Cancer Secreted Protein-2. Adv Sci (Weinh) 6:1802115. 10.1002/advs.201802115

Ji Q, Cai G, Liu X, Zhang Y, Wang Y, Zhou L, Sui H, and Li Q. 2019. MALAT1 regulates the transcriptional and translational levels of proto-oncogene RUNX2 in colorectal cancer metastasis. Cell Death Dis 10:378. 10.1038/s41419-019-1598-x

Jonas K, Calin GA, and Pichler M. 2020. RNA-Binding Proteins as Important Regulators of Long NonCoding RNAs in Cancer. Int J Mol Sci 21. 10.3390/ijms21082969

PeerJ reviewing PDF | (2020:11:55733:1:1:NEW 25 Feb 2021) 
481

482

483

484

485

486

487

488

489

490

491

492

493

494

495

496

497

498

499

500

501

502

503

504

505

506

507

508

509

510

511

512

513

Jung G, Hernández-Illán E, Moreira L, Balaguer F, and Goel A. 2020. Epigenetics of colorectal cancer: biomarker and therapeutic potential. Nat Rev Gastroenterol Hepatol 17:111-130. 10.1038/s41575-019-0230-y

Kim T, Jeon YJ, Cui R, Lee JH, Peng Y, Kim SH, Tili E, Alder H, and Croce CM. 2015. Role of MYCregulated long noncoding RNAs in cell cycle regulation and tumorigenesis. J Natl Cancer Inst 107. 10.1093/jnci/dju505

Kong J, and Lasko P. 2012. Translational control in cellular and developmental processes. Nat Rev Genet 13:383-394. 10.1038/nrg3184

Kong J, and Wang W. 2020. A Systemic Review on the Regulatory Roles of miR-34a in Gastrointestinal Cancer. Onco Targets Ther 13:2855-2872. 10.2147/ott.S234549

Konishi H, Fujiya M, Kashima S, Sakatani A, Dokoshi T, Ando K, Ueno N, Iwama T, Moriichi K, Tanaka H, and Okumura T. 2020. A tumor-specific modulation of heterogeneous ribonucleoprotein A0 promotes excessive mitosis and growth in colorectal cancer cells. Cell Death Dis 11:245. 10.1038/s41419-020-2439-7

Lafitte M, Sirvent A, and Roche S. 2020. Collagen Kinase Receptors as Potential Therapeutic Targets in Metastatic Colon Cancer. Front Oncol 10:125. 10.3389/fonc.2020.00125

Lew QJ, Chia YL, Chu KL, Lam YT, Gurumurthy M, Xu S, Lam KP, Cheong N, and Chao SH. 2012. Identification of HEXIM1 as a positive regulator of p53. J Biol Chem 287:36443-36454. 10.1074/jbc.M112.374157

Liang W, Zhang L, Jiang G, Wang Q, Liu L, Liu D, Wang Z, Zhu Z, Deng Q, Xiong X, Shao W, Shi X, and He J. 2015. Development and validation of a nomogram for predicting survival in patients with resected non-small-cell lung cancer. J Clin Oncol 33:861-869. 10.1200/jco.2014.56.6661

Lin J, Chen Y, Tang WF, Liu C, Zhang S, Guo ZQ, Chen G, and Zheng XW. 2019. PPARG rs3856806 C $>$ T Polymorphism Increased the Risk of Colorectal Cancer: A Case-Control Study in Eastern Chinese Han Population. Front Oncol 9:63. 10.3389/fonc.2019.00063

Liu Y, Wu L, Ao H, Zhao M, Leng X, Liu M, Ma J, and Zhu J. 2019. Prognostic implications of autophagy-associated gene signatures in non-small cell lung cancer. Aging (Albany NY) 11. 10.18632/aging. 102544

Lukong KE, Chang KW, Khandjian EW, and Richard S. 2008. RNA-binding proteins in human genetic disease. Trends Genet 24:416-425. 10.1016/j.tig.2008.05.004

Luo H, Xu G, Li C, He L, Luo L, Wang Z, Jing B, Deng Y, Jin Y, Li Y, Li B, Tan W, He C, Seeruttun SR, Wu Q, Huang J, Huang DW, Chen B, Lin SB, Chen QM, Yuan CM, Chen HX, Pu HY, Zhou F, He Y, and Xu RH. 2019. Real-time artificial intelligence for detection of upper gastrointestinal 
514

515

516

517

518

519

520

521

522

523

524

525

526

527

528

529

530

531

532

533

534

535

536

537

538

539

540

541

542

543

544

545

546

cancer by endoscopy: a multicentre, case-control, diagnostic study. Lancet Oncol 20:1645-1654. 10.1016/s1470-2045(19)30637-0

Ma S, Long T, and Huang WJM. 2019. Noncoding RNAs in inflammation and colorectal cancer. RNA Biol:1-8. 10.1080/15476286.2019.1705610

Marisa L, de Reyniès A, Duval A, Selves J, Gaub MP, Vescovo L, Etienne-Grimaldi MC, Schiappa R, Guenot D, Ayadi M, Kirzin S, Chazal M, Fléjou JF, Benchimol D, Berger A, Lagarde A, Pencreach E, Piard F, Elias D, Parc Y, Olschwang S, Milano G, Laurent-Puig P, and Boige V. 2013. Gene expression classification of colon cancer into molecular subtypes: characterization, validation, and prognostic value. PLoS Med 10:e1001453. 10.1371/journal.pmed.1001453

Miao Y, Li Q, Wang J, Quan W, Li C, Yang Y, and Mi D. 2020. Prognostic implications of metabolismassociated gene signatures in colorectal cancer. PeerJ 8:e9847. 10.7717/peerj.9847

Mitobe Y, Iino K, Takayama KI, Ikeda K, Suzuki T, Aogi K, Kawabata H, Suzuki Y, Horie-Inoue K, and Inoue S. 2020. PSF promotes ER-positive breast cancer progression via posttranscriptional regulation of ESR1 and SCFD2. Cancer Res. 10.1158/0008-5472.Can-19-3095

Mo S, Dai W, Xiang W, Li Y, Feng Y, Zhang L, Li Q, and Cai G. 2019. Prognostic and predictive value of an autophagy-related signature for early relapse in stages I-III colon cancer. Carcinogenesis 40:861-870. 10.1093/carcin/bgz031

Morak M, Koehler U, Schackert HK, Steinke V, Royer-Pokora B, Schulmann K, Kloor M, Höchter W, Weingart J, Keiling C, Massdorf T, and Holinski-Feder E. 2011. Biallelic MLH1 SNP cDNA expression or constitutional promoter methylation can hide genomic rearrangements causing Lynch syndrome. J Med Genet 48:513-519. 10.1136/jmedgenet-2011-100050

Murphy N, Jenab M, and Gunter MJ. 2018. Adiposity and gastrointestinal cancers: epidemiology, mechanisms and future directions. Nat Rev Gastroenterol Hepatol 15:659-670. 10.1038/s41575018-0038-1

Noubissi FK, Elcheva I, Bhatia N, Shakoori A, Ougolkov A, Liu J, Minamoto T, Ross J, Fuchs SY, and Spiegelman VS. 2006. CRD-BP mediates stabilization of betaTrCP1 and c-myc mRNA in response to beta-catenin signalling. Nature 441:898-901. 10.1038/nature04839

Qi J, Yu Y, Akilli Öztürk Ö, Holland JD, Besser D, Fritzmann J, Wulf-Goldenberg A, Eckert K, Fichtner , and Birchmeier W. 2016. New Wnt/ $\beta$-catenin target genes promote experimental metastasis and migration of colorectal cancer cells through different signals. Gut 65:1690-1701. 10.1136/gutjnl2014-307900

Reichling C, Taieb J, Derangere V, Klopfenstein Q, Le Malicot K, Gornet JM, Becheur H, Fein F, Cojocarasu O, Kaminsky MC, Lagasse JP, Luet D, Nguyen S, Etienne PL, Gasmi M, Vanoli A, 
547

548

549

550

551

552

553

554

555

556

557

558

559

560

561

562

563

564

565

566

567

568

569

570

571

572

573

574

575

576

577

578
Perrier H, Puig PL, Emile JF, Lepage C, and Ghiringhelli F. 2019. Artificial intelligence-guided tissue analysis combined with immune infiltrate assessment predicts stage III colon cancer outcomes in PETACC08 study. Gut. 10.1136/gutjnl-2019-319292

Ritchie ME, Phipson B, Wu D, Hu Y, Law CW, Shi W, and Smyth GK. 2015. limma powers differential expression analyses for RNA-sequencing and microarray studies. Nucleic Acids Research 43:e47e47. 10.1093/nar/gkv007 \%J Nucleic Acids Research

Rokavec M, Kaller M, Horst D, and Hermeking H. 2017. Pan-cancer EMT-signature identifies RBM47 down-regulation during colorectal cancer progression. Sci Rep 7:4687. 10.1038/s41598-01704234-2

Sachs MC. 2017. plotROC: A Tool for Plotting ROC Curves. J Stat Softw 79. 10.18637/jss.v079.c02

Sharma R. 2020. An examination of colorectal cancer burden by socioeconomic status: evidence from GLOBOCAN 2018. Epma j 11:95-117. 10.1007/s13167-019-00185-y

Sjoquist KM, Renfro LA, Simes RJ, Tebbutt NC, Clarke S, Seymour MT, Adams R, Maughan TS, Saltz L, Goldberg RM, Schmoll HJ, Van Cutsem E, Douillard JY, Hoff PM, Hecht JR, Tournigand C, Punt CJA, Koopman M, Hurwitz H, Heinemann V, Falcone A, Porschen R, Fuchs C, Diaz-Rubio E, Aranda E, Bokemeyer C, Souglakos I, Kabbinavar FF, Chibaudel B, Meyers JP, Sargent DJ, de Gramont A, and Zalcberg JR. 2018. Personalizing Survival Predictions in Advanced Colorectal Cancer: The ARCAD Nomogram Project. J Natl Cancer Inst 110:638-648. $10.1093 /$ jnci/djx253

van Kouwenhove M, Kedde M, and Agami R. 2011. MicroRNA regulation by RNA-binding proteins and its implications for cancer. Nat Rev Cancer 11:644-656. 10.1038/nrc3107

von Mering C, Huynen M, Jaeggi D, Schmidt S, Bork P, and Snel B. 2003. STRING: a database of predicted functional associations between proteins. Nucleic Acids Res 31:258-261. $10.1093 / \mathrm{nar} / \mathrm{gkg} 034$

Waku T, Katayama H, Hiraoka M, Hatanaka A, Nakamura N, Tanaka Y, Tamura N, Watanabe A, and Kobayashi A. 2020. NFE2L1 and NFE2L3 complementarily maintain basal proteasome activity in cancer cells through CPEB3-mediated translational repression. Mol Cell Biol. 10.1128/mcb.00010-20

Wang E, Lu SX, Pastore A, Chen X, Imig J, Chun-Wei Lee S, Hockemeyer K, Ghebrechristos YE, Yoshimi A, Inoue D, Ki M, Cho H, Bitner L, Kloetgen A, Lin KT, Uehara T, Owa T, Tibes R, Krainer AR, Abdel-Wahab O, and Aifantis I. 2019a. Targeting an RNA-Binding Protein Network in Acute Myeloid Leukemia. Cancer Cell 35:369-384.e367. 10.1016/j.ccell.2019.01.010

Peer) reviewing PDF | (2020:11:55733:1:1:NEW 25 Feb 2021) 
579 Wang L, Cho KB, Li Y, Tao G, Xie Z, and Guo B. 2019b. Long Noncoding RNA (lncRNA)-Mediated

580

581

582

583

584

585

586

587

588

589

590

591

592

593

594

595

596

597

598

599

600

601

602

603

604

605
Competing Endogenous RNA Networks Provide Novel Potential Biomarkers and Therapeutic Targets for Colorectal Cancer. Int J Mol Sci 20. 10.3390/ijms20225758

Won YW, Joo J, Yun T, Lee GK, Han JY, Kim HT, Lee JS, Kim MS, Lee JM, Lee HS, Zo JI, and Kim S. 2015. A nomogram to predict brain metastasis as the first relapse in curatively resected non-small cell lung cancer patients. Lung Cancer 88:201-207. 10.1016/j.lungcan.2015.02.006

Wu XL, Yang ZW, He L, Dong PD, Hou MX, Meng XK, Zhao HP, Wang ZY, Wang F, Baoluri, Wurenqimuge, Agudamu, Jia YF, and Shi L. 2017. RRS1 silencing suppresses colorectal cancer cell proliferation and tumorigenesis by inhibiting G2/M progression and angiogenesis. Oncotarget 8:82968-82980. 10.18632/oncotarget.20897

Xiong Y, Wang R, Peng L, You W, Wei J, Zhang S, Wu X, Guo J, Xu J, Lv Z, and Fu Z. 2017. An integrated lncRNA, microRNA and mRNA signature to improve prognosis prediction of colorectal cancer. Oncotarget 8:85463-85478. 10.18632/oncotarget.20013

Zhou B, and Guo R. 2018. Integrative analysis of significant RNA-binding proteins in colorectal cancer metastasis. J Cell Biochem 119:9730-9741. 10.1002/jcb.27290.

Zhang S, Wu Z, Xie J, Yang Y, Wang L, and Qiu H. 2019. DNA methylation exploration for ARDS: a multi-omics and multi-microarray interrelated analysis. J Transl Med 17:345. 10.1186/s12967019-2090-1

Zhang Z, Wang L, Wang Q, Zhang M, Wang B, Jiang K, Ye Y, Wang S, and Shen Z. 2020. Molecular Characterization and Clinical Relevance of RNA Binding Proteins in Colorectal Cancer. Front Genet 11:580149. 10.3389/fgene.2020.580149

Zhu S, Rooney S, and Michlewski G. 2020. RNA-Targeted Therapies and High-Throughput Screening Methods. Int J Mol Sci 21. 10.3390/ijms21082996 
Figure 1

Figure 1. A flow chart of the study design and analysis. 


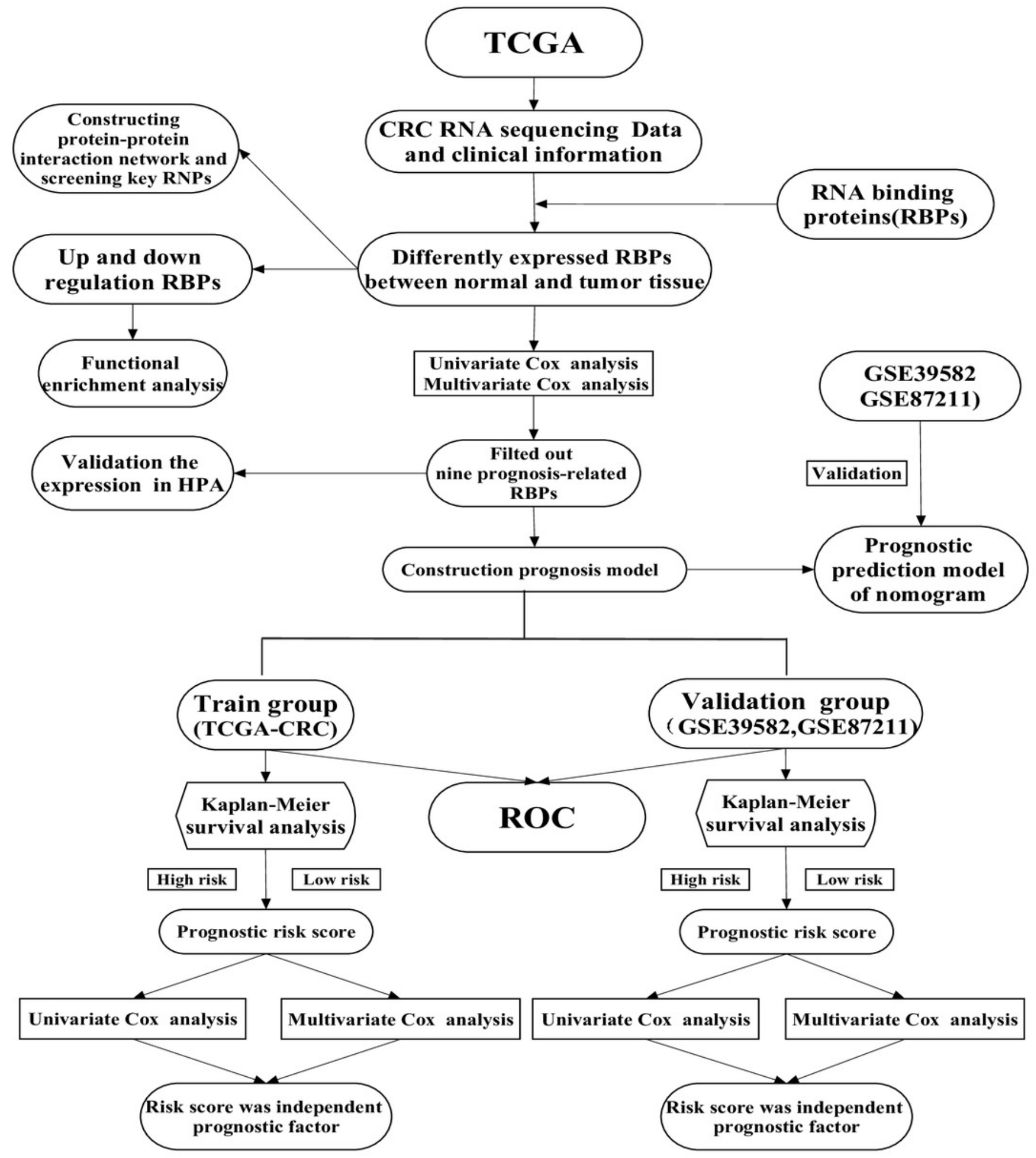




\section{Figure 2}

Figure 2. Differentially expressed RBPs in Colorectal cancer.

(A) Heatmap: higher expression RBPs are shown in red; lower expressions RBPs are shown in green. (B)Volcano diagram: Each point represents a protein: down-regulated (blue), upregulated (red), and no significant (black). RBPs, RNA-binding proteins.
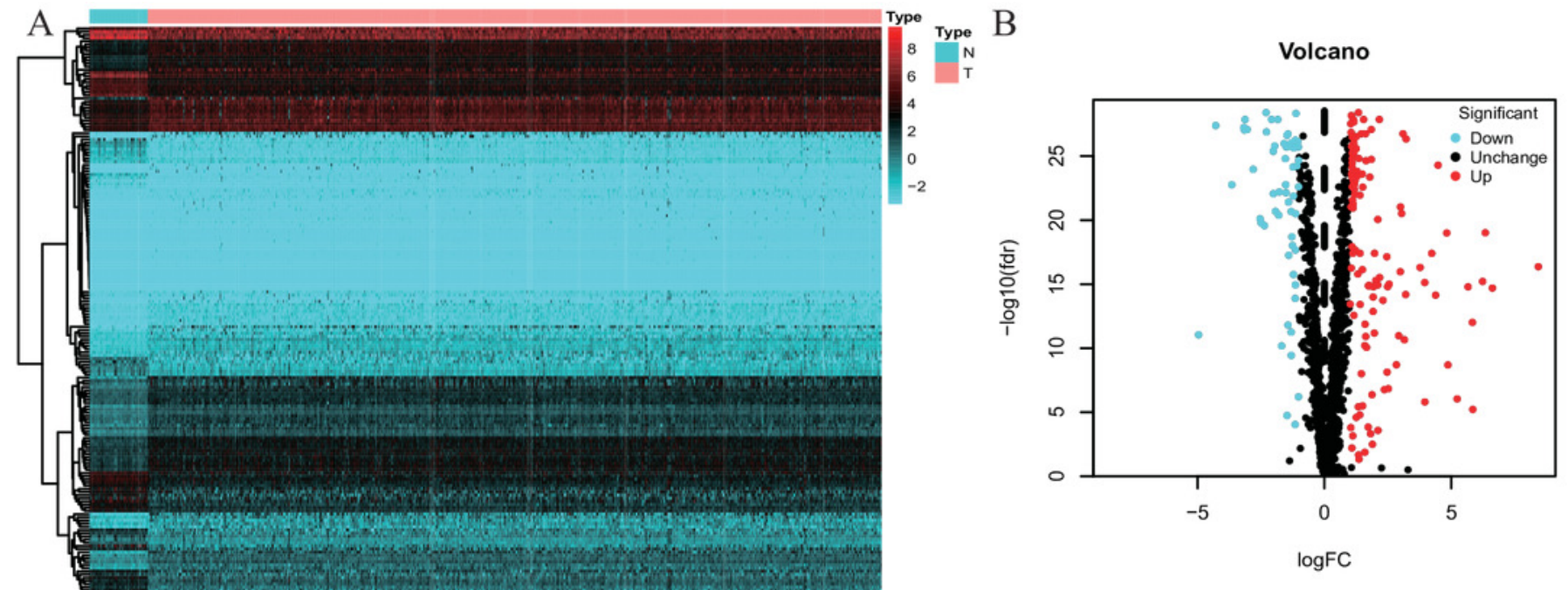
Figure 3

Figure 3. Functional enrichment analysis of RBPs.

(A) TOP-10 terms of BP, CC, and MF in up-regulated RBPs. (B) TOP-10 terms of BP, CC, and MF in down-regulated RBPs. (C-L) TOP-5 GSEA analysis of the KEGG pathway in RBPs. RBPs, RNA-binding proteins. BP, Biological Processes, CC, Cellular Components, MF, Molecular Functions, KEGG, Kyoto Encyclopedia of Genes and Genomes, GSEA, Gene Set Enrichment Analysis.

A

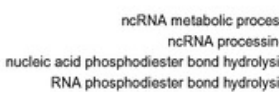
RNA phosphodiostor bond hydrolysis

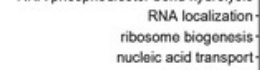

nucloic acid transport RNA transport
RNA localization
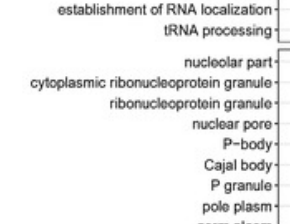

germ plasm
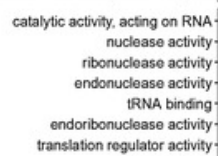

translation rogulator activity pseudouridine synthese activity

C

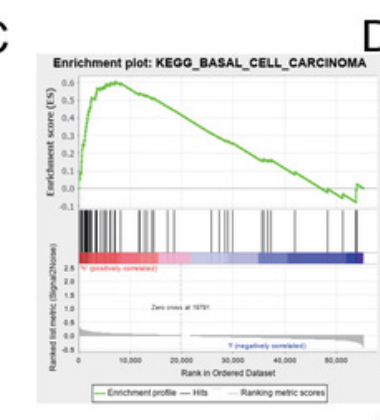

$\mathrm{H}$

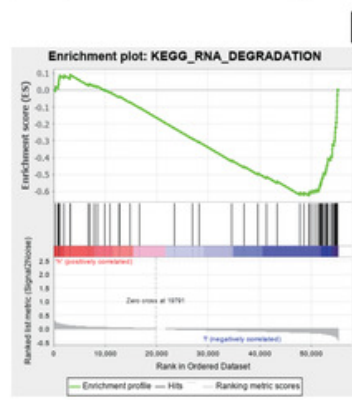

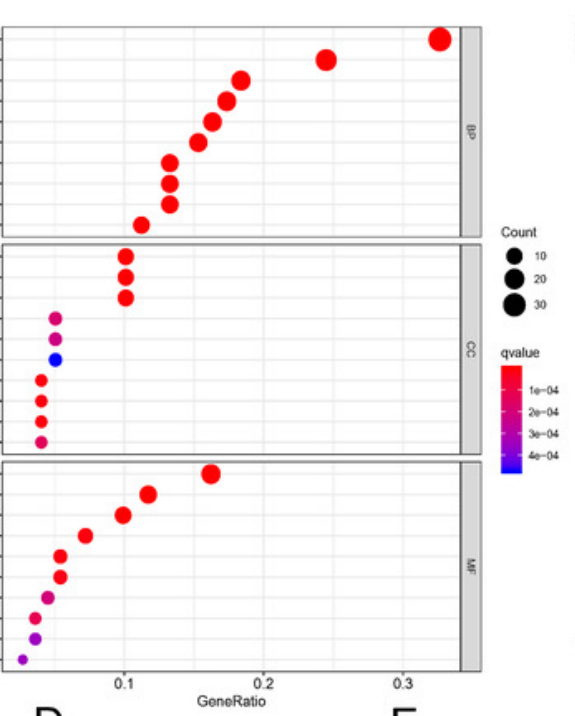

E
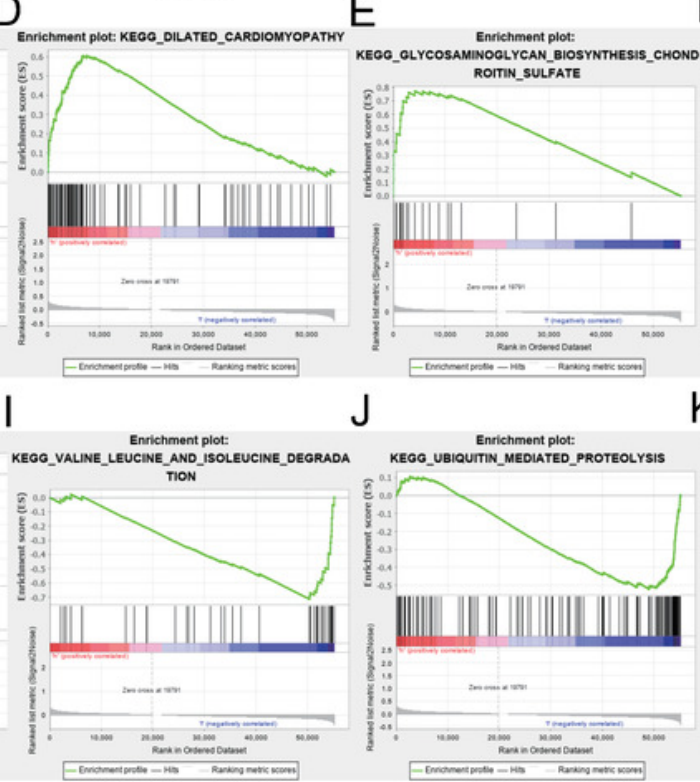

B

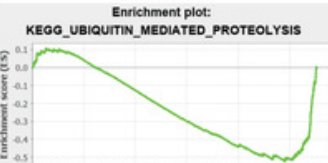

\|\|\|\|\|\|\|\|\|\|\|\|\|\|\|\|$\|$
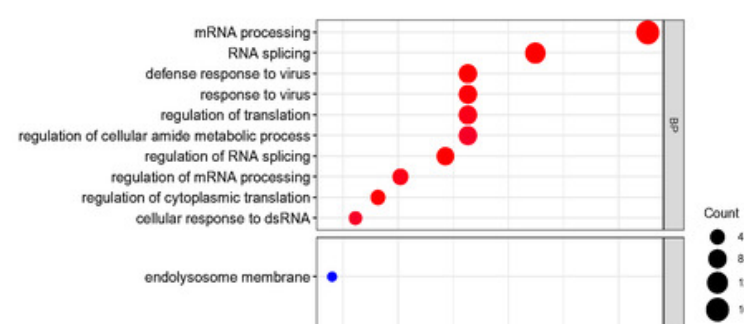

single-stranded RNA binding

translation regulator activity, nucleic acid binding

$\mathrm{F}$
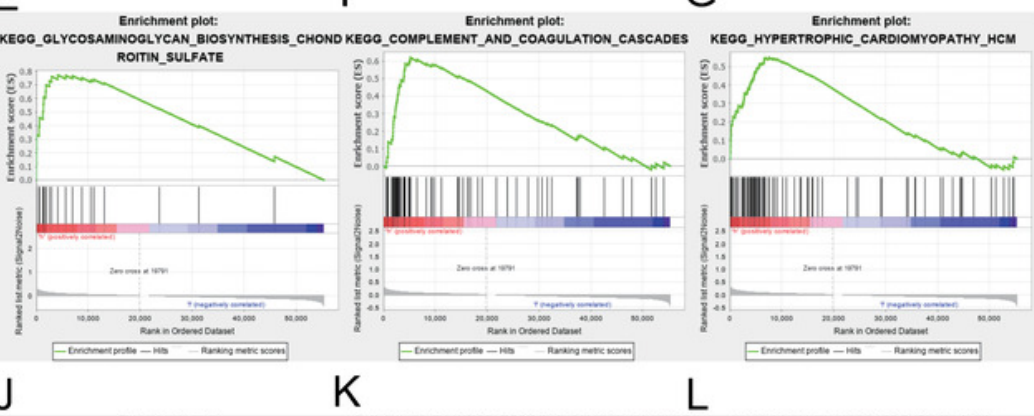

$\mathrm{L}$
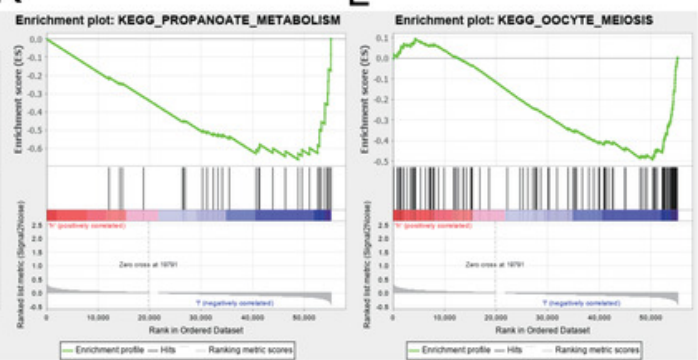
Figure 4

Figure 4. Protein-protein interaction network and module analysis.

(A) Protein-protein interaction (PPI) network of differentially expressed RBPs. (B) Critical module from the PPI network. Blue circles: down-regulation RBPs (adjust P-value $\square 0.05$ ), red circles: upregulation RBPs(adjust P-value[0.05). RBPs, RNA-binding proteins.

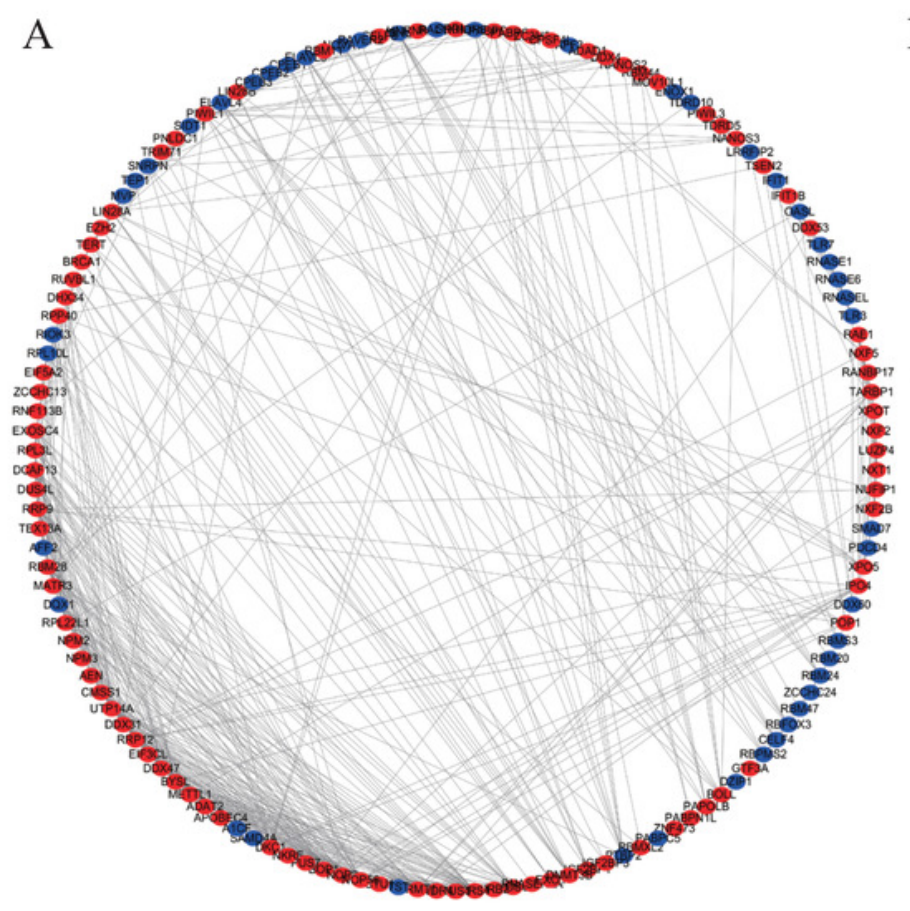

B

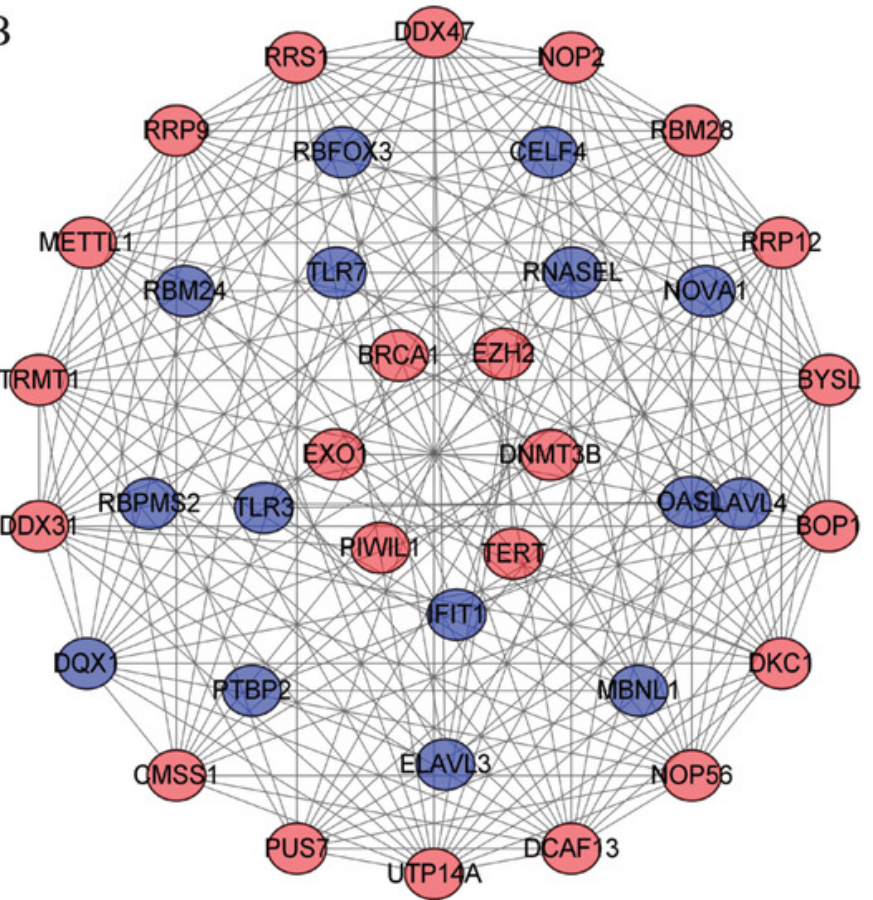




\section{Figure 5}

Figure 5. Risk score analysis of nine-RBPs prognostic model in the TCGA cohort.

(A) The hazard ratios calculated by univariate Cox regression. (B) The hazard ratios calculated by multivariate Cox regression analysis. (C) Kaplan-Meier survival analysis of CRC patients for high and low risk subgroups. (D) ROC analysis of the specificity and sensitivity of the OS based on the risk score. (E) Heatmap of the BAPs expression profiles. $(\mathbf{F}, \mathbf{G})$ The distribution of patient's survival time and survival status. The black dotted line is the optimum cutoff dividing patients into high risk and low risk groups. (H) Univariate independent prognosis Cox regression analysis. Forest plot of the relationship between risk factors and OS. (I) Multivariate independent prognosis Cox regression analysis. Forest plot of the association between risk factors and OS. ROC, Receiver Operating Characteristic, OS, Overall survival. 
A $\begin{array}{lrr} & \text { pvalue } & \begin{array}{r}\text { Hazard ratio } \\ \text { ZC3H12C }\end{array} \\ \text { O.020 } & 0.807(0.674-0.967) \\ \text { PNLDC1 } & 0.031 & 1.227(1.019-1.476) \\ \text { LUZP4 } & <0.001 & 1.531(1.246-1.882) \\ \text { RRS1 } & 0.011 & 1.484(1.095-2.011) \\ \text { HEXIM1 } & 0.038 & 1.371(1.018-1.847) \\ \text { SRRM3 } & 0.037 & 1.659(1.031-2.671) \\ \text { PPARGC1A } & 0.046 & 0.809(0.657-0.996) \\ \text { PPARGC1B } & 0.006 & 0.594(0.410-0.862) \\ \text { RBPMS2 } & 0.040 & 1.310(1.013-1.695) \\ \text { BRCA1 } & 0.028 & 0.653(0.447-0.955) \\ \text { CELF4 } & <0.001 & 1.676(1.265-2.220) \\ \text { AEN } & 0.023 & 1.351(1.043-1.752) \\ \text { LRRFIP2 } & 0.043 & 0.707(0.506-0.989) \\ \text { EIF4E3 } & 0.033 & 0.675(0.471-0.969) \\ \text { NOVA1 } & 0.002 & 1.327(1.108-1.589)\end{array}$

$\mathrm{C}$

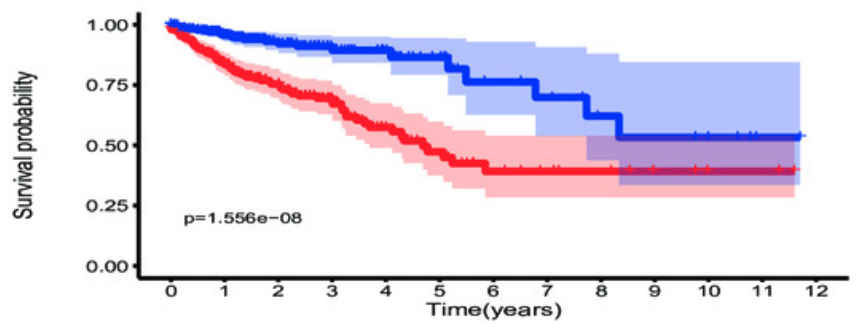

\begin{tabular}{l|ccccccccccccc}
.丷. High risk & 293 & 178 & 101 & 59 & 33 & 20 & 12 & 9 & 7 & 4 & 2 & 2 & 0 \\
\hline ․ Low risk & 293 & 194 & 99 & 56 & 32 & 21 & 13 & 11 & 7 & 6 & 4 & 1 & 0
\end{tabular} × Low risk \begin{tabular}{ccccccccccccc}
293 & 194 & 99 & 56 & 32 & 21 & 13 & 11 & 7 & 6 & 4 & 1 & 0 \\
\hline 0 & 1 & 2 & 3 & 4 & 5 & 6 & 7 & 8 & 9 & 10 & 11 & 12
\end{tabular} $\mathrm{E}$

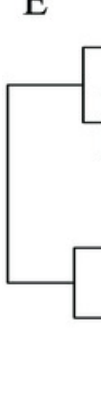

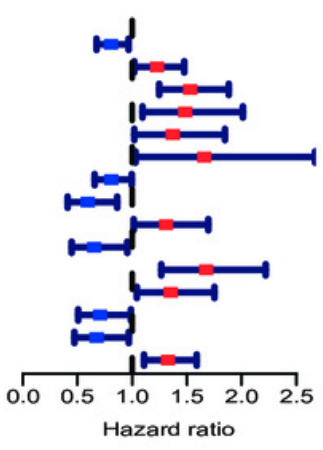

D

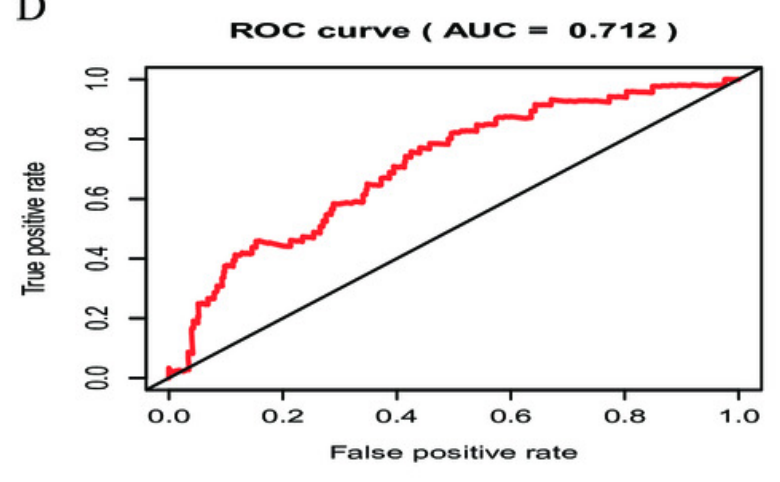

B

$\begin{array}{lrr} & \text { pvalue } & \text { Hazard ratio } \\ \text { PNLDC1 } & 0.005 & 1.342(1.094-1.647) \\ \text { RRS1 } & 0.004 & 1.622(1.171-2.247) \\ \text { HEXIM1 } & 0.026 & 1.435(1.045-1.969) \\ \text { PPARGC1A } & 0.128 & 0.839(0.669-1.052) \\ \text { PPARGC1B } & 0.074 & 0.693(0.464-1.036) \\ \text { BRCA1 } & 0.059 & 0.660(0.428-1.016) \\ \text { CELF4 } & <0.001 & 1.726(1.307-2.280) \\ \text { AEN } & 0.002 & 1.549(1.169-2.052) \\ \text { NOVA1 } & 0.121 & 1.178(0.958-1.449)\end{array}$

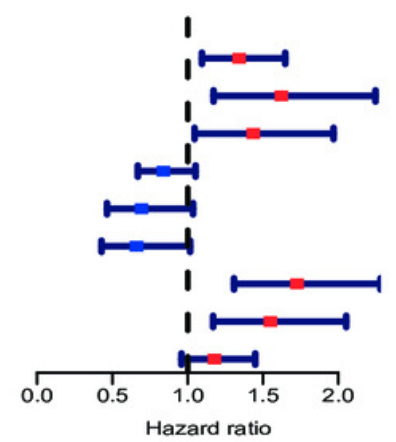

$\mathrm{F}$

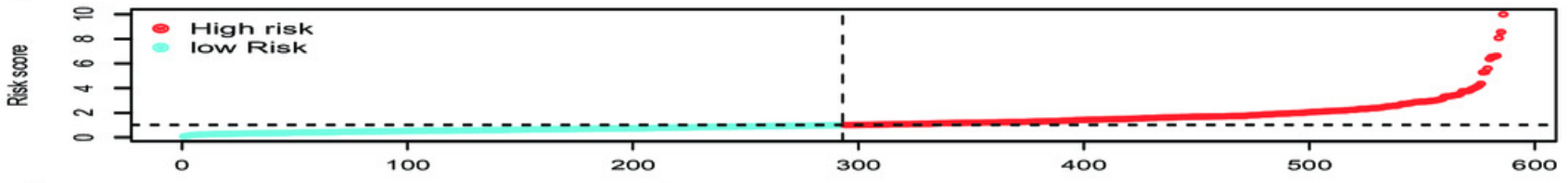

$\mathrm{G}$

Patients (increasing risk socre)

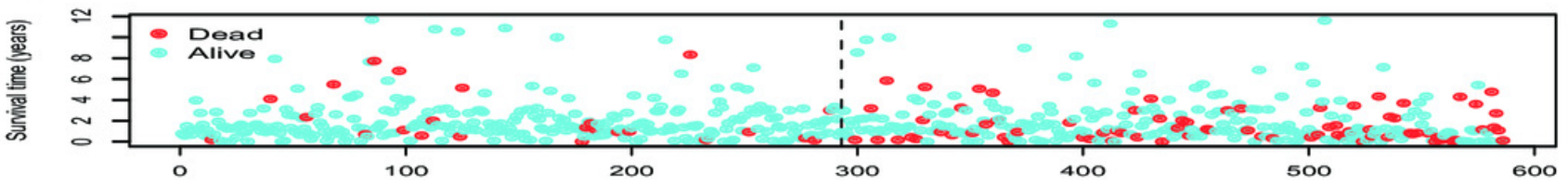

$\mathrm{H}$

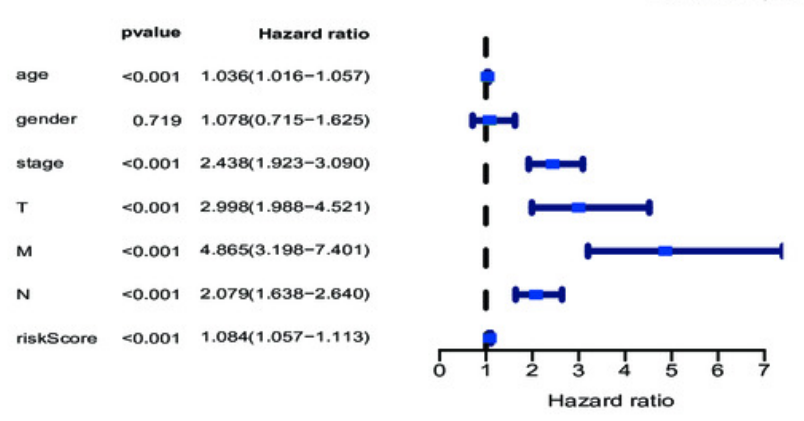

I
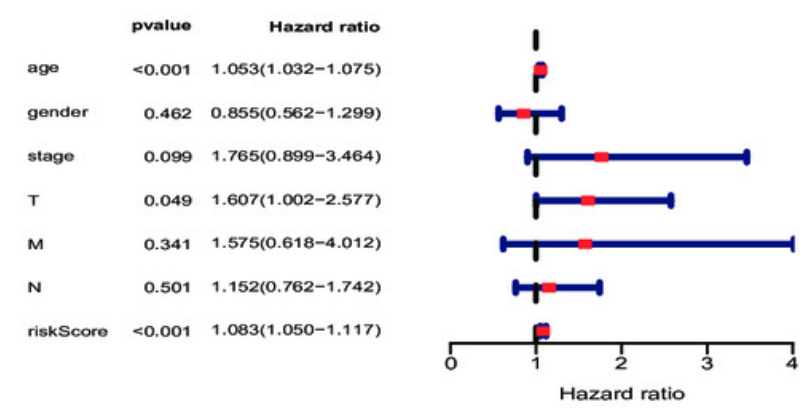


\section{Figure 6}

Figure 6. Risk score analysis of nine-RBPs prognostic model in the GEO cohort.

(A) Kaplan-Meier survival analysis of CRC patients for high and low risk subgroups. (B) ROC analysis of the specificity and sensitivity of the OS based on the risk score. (C) Heatmap of the RBPs expression profiles. (D, E) The distribution of patient's survival time and survival status. The black dotted line is the optimum cutoff dividing patients into high risk and low risk groups. (F) Univariate independent prognosis Cox regression analysis. Forest plot of the relationship between risk factors and OS. (G) Multivariate independent prognosis Cox regression analysis. Forest plot of the association between risk factors and OS. ROC, Receiver Operating Characteristic, OS, Overall survival. 
A

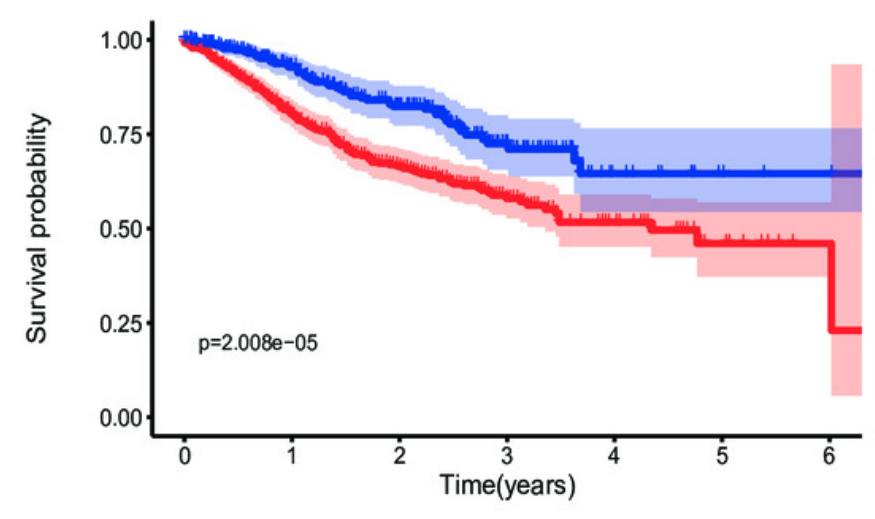

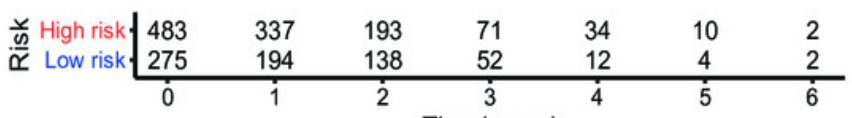

C
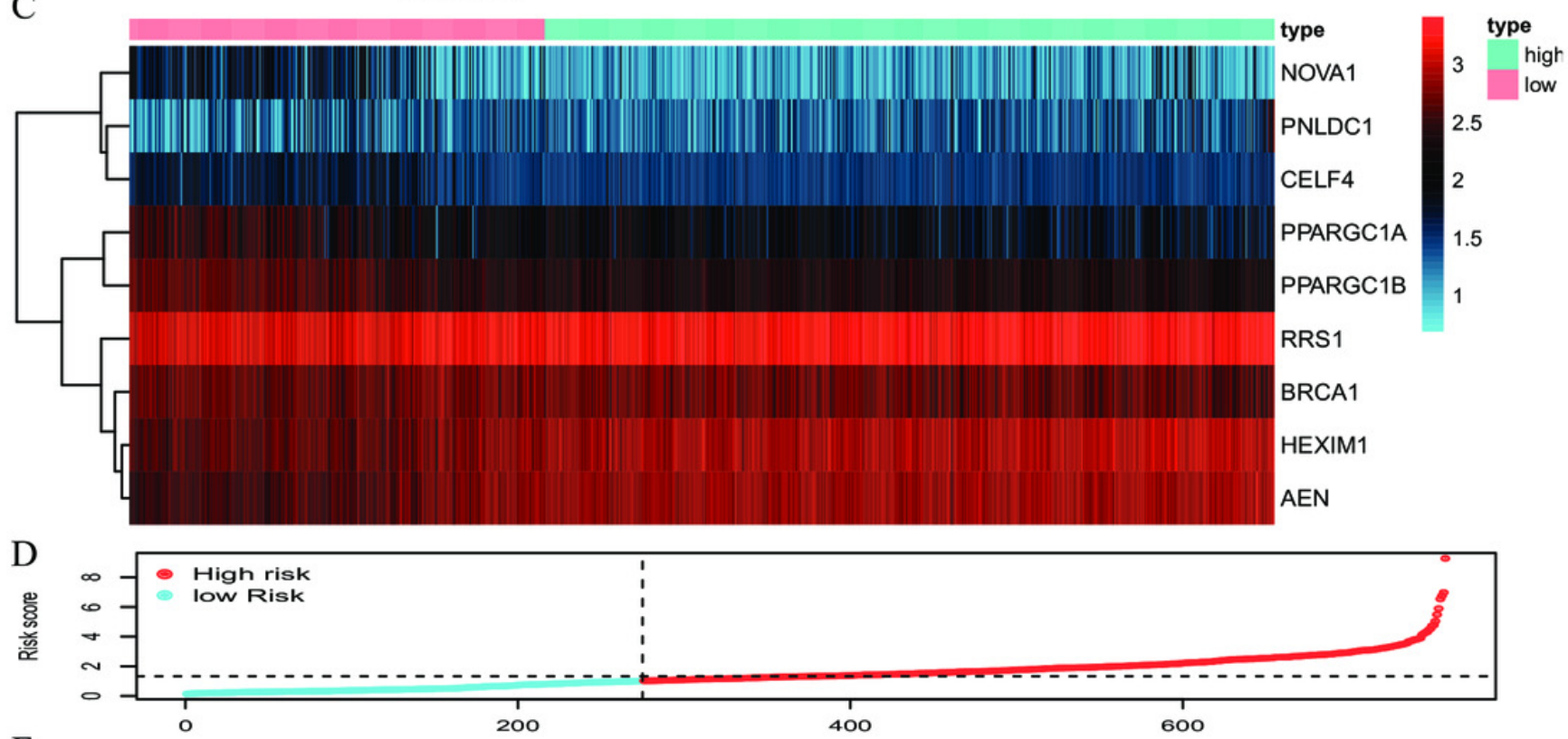

E

$$
\text { 爱 }
$$

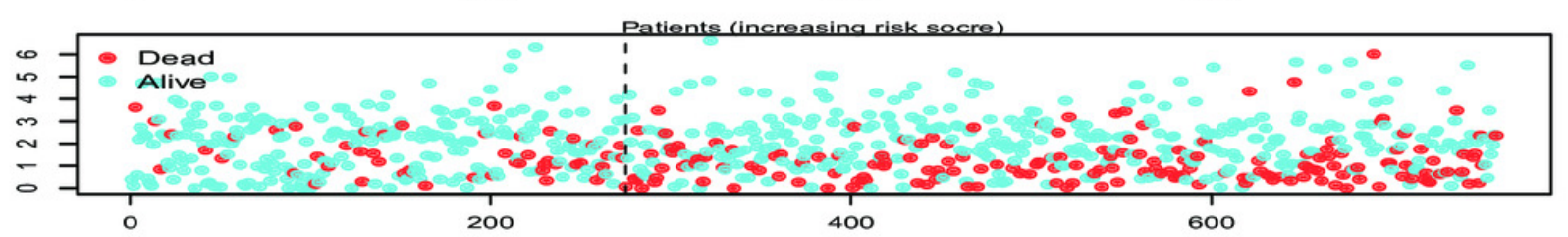

F
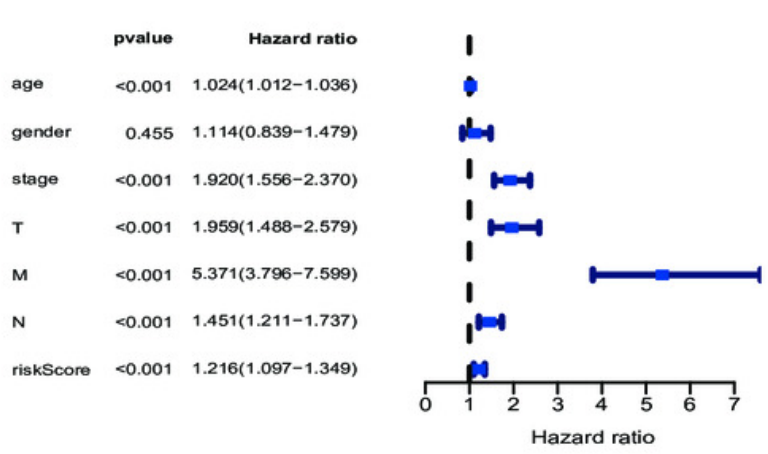

$\mathrm{G}$

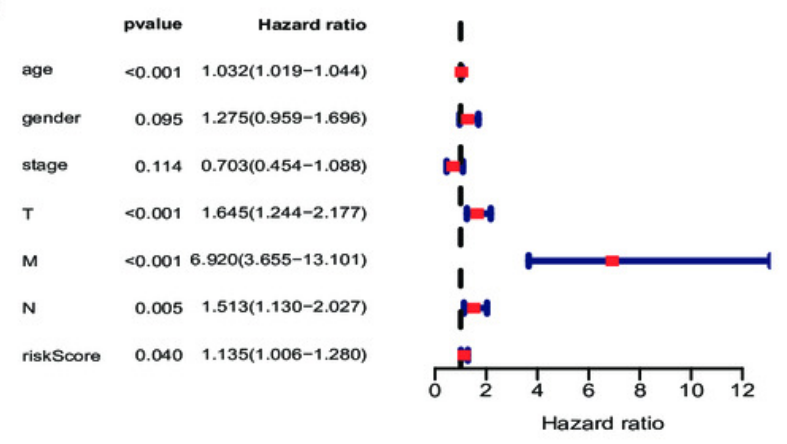


Figure 7

Figure 7. The nomogram to predict 1-, 3-, and 5-year OS of CRC patients.

(A) The nomogram for predicting 1-, 3- and 5-year OS of CRC by nine RBPs signature. The Calibration curves for predicting 1-, 3- and 5-year OS of TCGA-CRC (B, D, F) and GEO-CRC (C, E, G). OS, Overall survival. 
A

Points

PNLDC1

RRS1

HEXIM1

PPARGC1A

PPARGC1B

BRCA1

CELF4

AEN

NOVA1

Total Points

1-year survival

3-year survival

5-year survival

B

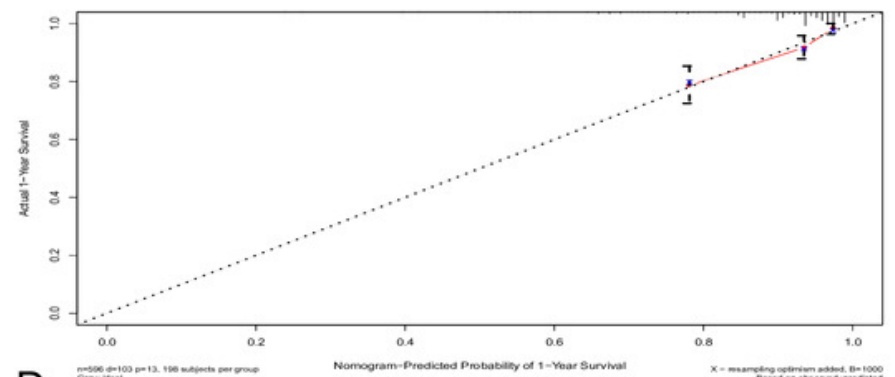

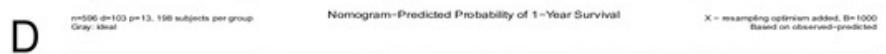
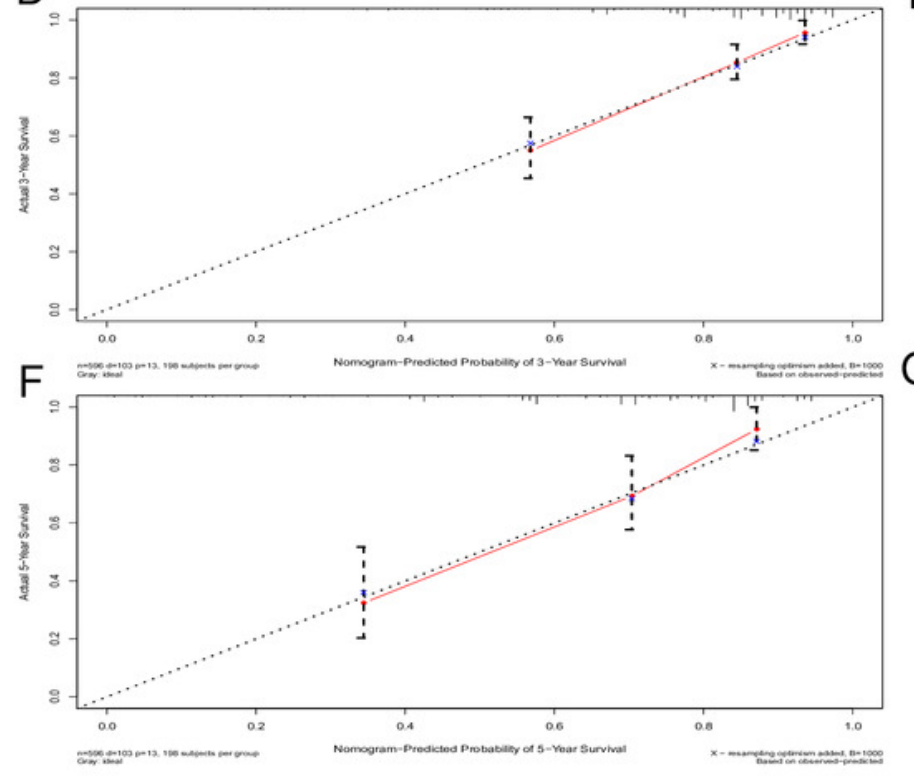

$\begin{array}{llllll}7.5 & 6 & 5 & 4 & 3 & 2\end{array}$

$\begin{array}{lllllllllll}7 & 1 & 3 & 1 & 5 & 1 & 1 & \\ 1 & 2 & 3 & 4 & 5 & 6 & 7 & 8\end{array}$

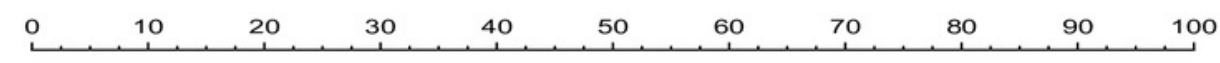

\begin{tabular}{|c|c|c|c|c|c|c|c|c|c|c|c|c|c|}
\hline 1 & 2 & 3 & & 4 & 5 & 6 & 7 & & 8 & 9 & 10 & 11 & 12 \\
\hline 5.5 & 6 & 6.5 & 7 & 7.5 & 8 & 8.5 & 9 & 9.5 & 10 & 10.5 & & & \\
\hline 4 & .5 & 5.5 & 6 & 6.5 & 7.5 & 8 & & & & & & & \\
\hline
\end{tabular}

$\begin{array}{lllllllll}6.5 & 6 & 5.5 & 5 & 4.5 & 4 & 3.5 & 3 & 2.5\end{array}$

$\begin{array}{lllllllll}7.5 & 7 & 6.5 & 6 & 5.5 & 5 & 4.5 & 4 & 3.5\end{array}$

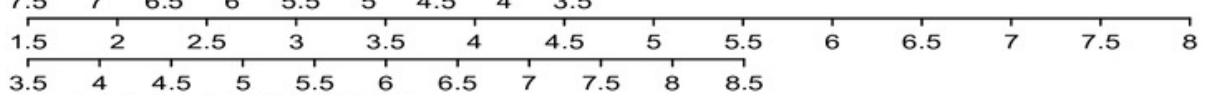

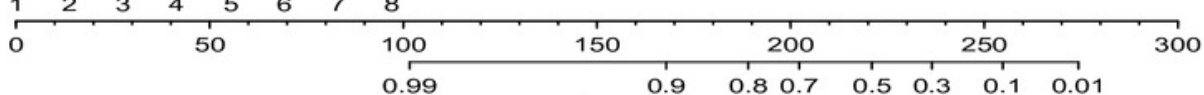

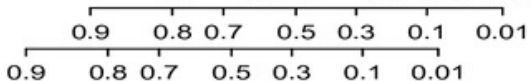

$\begin{array}{lllllll}0.9 & 0.8 & 0.7 & 0.5 & 0.3 & 0.1 & 0.01\end{array}$

C

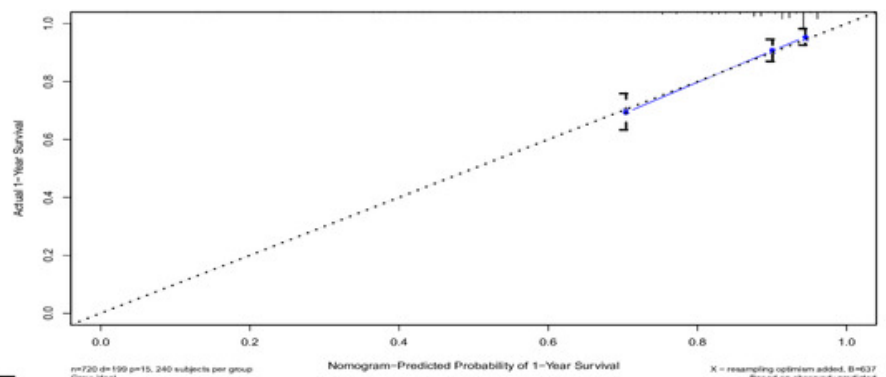

E
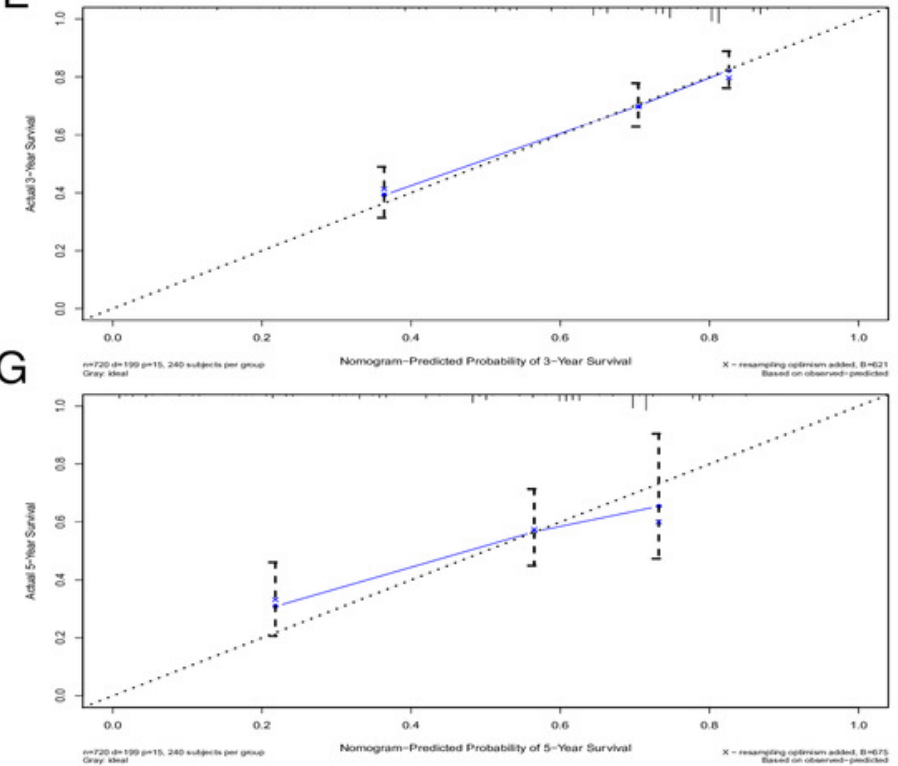
Figure 8

Figure 8. The Co-expression analysis of RBPs.

(A) The proteins in the left box are RBPs, and in the right box are co-expressed proteins with

RBPs. (B) CELF4 was positively co-expressed with LUZP4. NOVA1 was positively co-expressed with AFF3 (C) CPEB1 (D) DZIP1 (E) ELAVL4 (F) ENOX1 (G) RBFOX3(H) RBPMS2 (I) RBM20 (J) SAMD4A (K) ZCCHC24 (L). PPARGC1A was positively co-expressed with CPEB3 (M) RAVER2 (N) RBM47 (0). PPARGC1B was positively co-expressed with LRRFIP2 (P) RAVER2 (Q) RBM47 (R). RRS1 was positively co-expressed with BOP1 (S). 
A
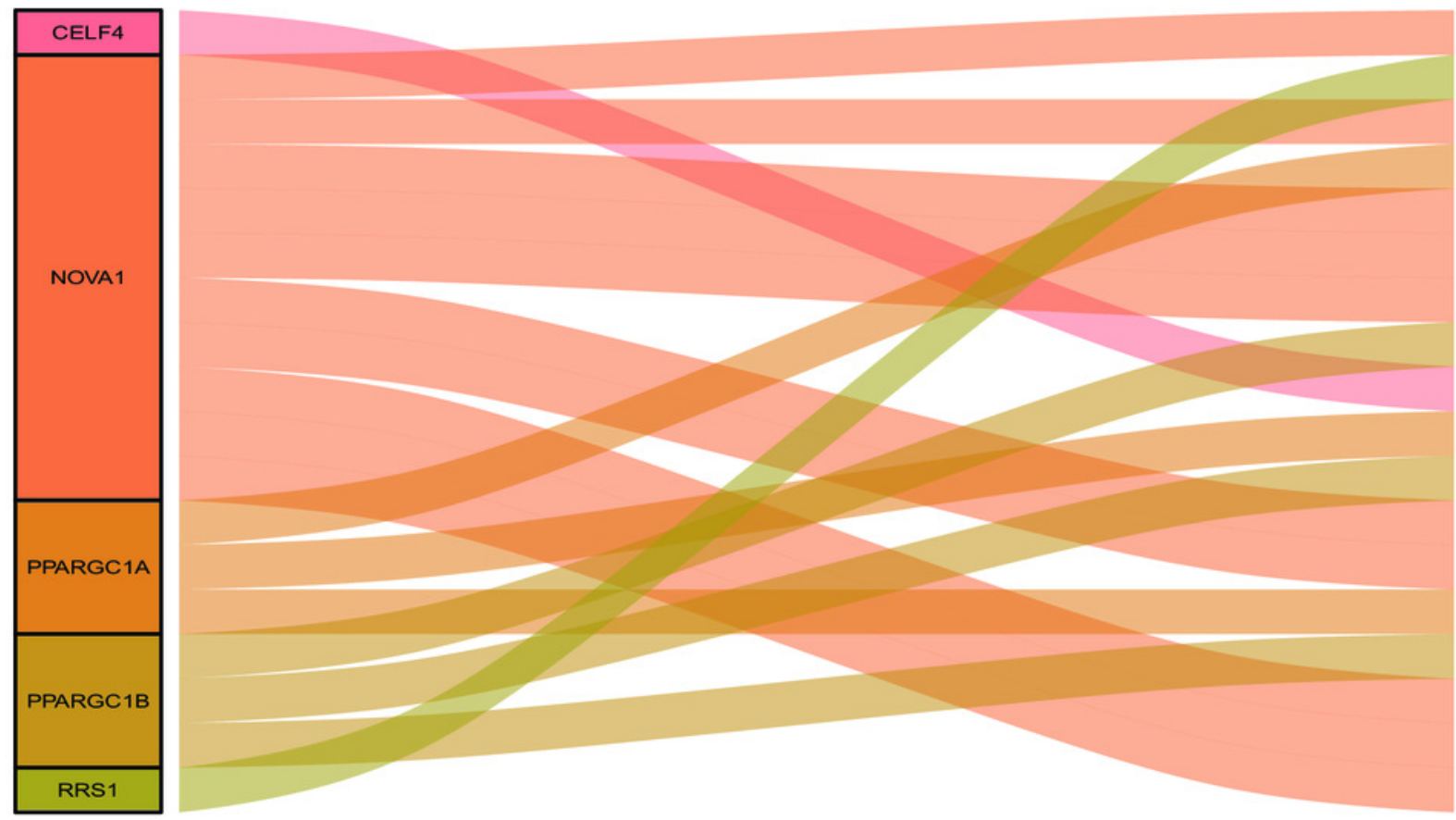

\begin{tabular}{|c|}
\hline AFF 3 \\
\hline BOP1 \\
\hline CPEB1 \\
\hline CPEB3 \\
\hline DZIP1 \\
\hline ELAVL4 \\
\hline ENOX1 \\
\hline LRRFIP2 \\
\hline LUZP4 \\
\hline RAVER2 \\
\hline RBFOX3 \\
\hline RBM20 \\
\hline RBM47 \\
\hline RBPMS2 \\
\hline SAMD4A \\
\hline ZCCHC24 \\
\hline
\end{tabular}
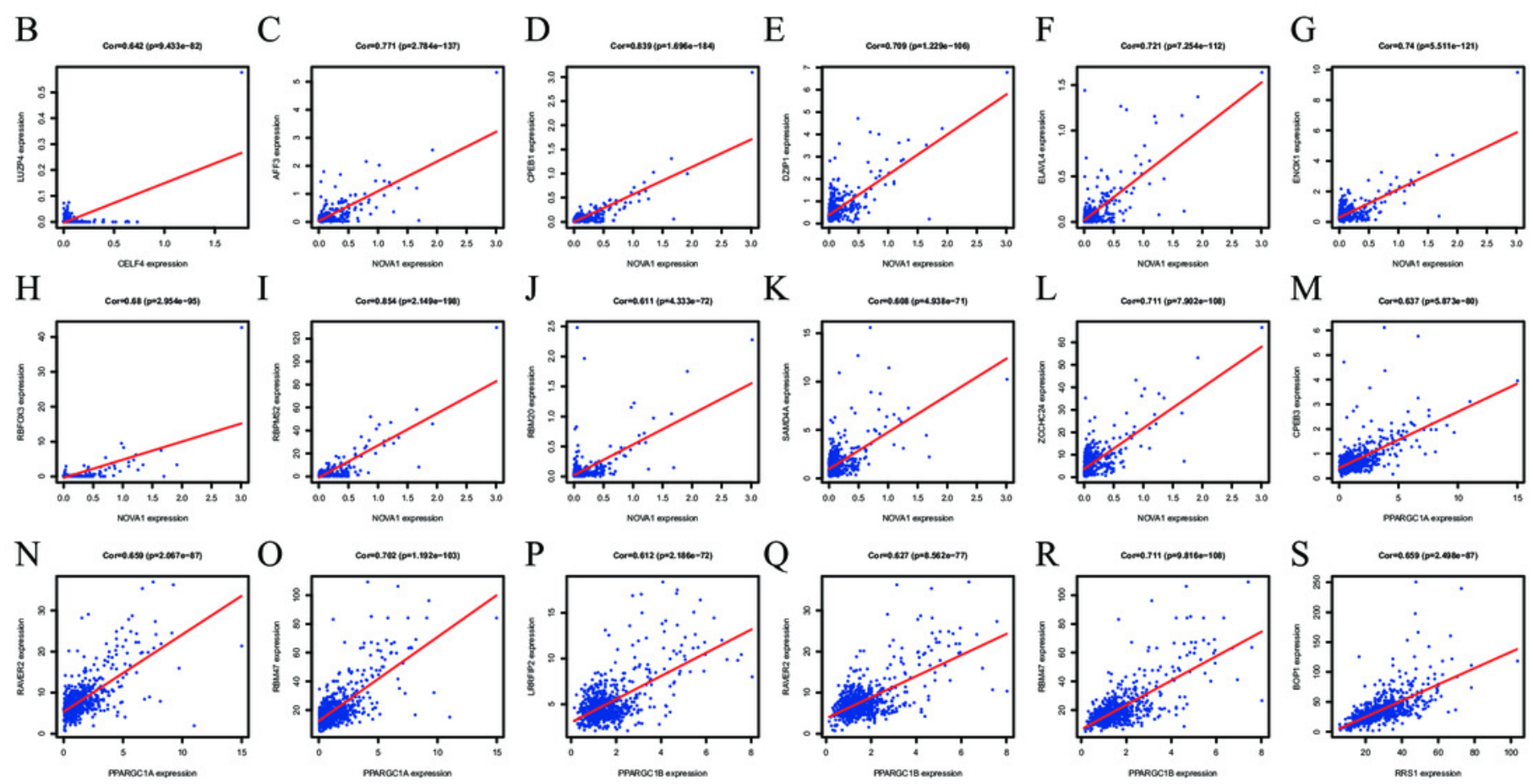
Figure 9

Figure 9. Validation of hub RBPs expression in CRC and normal colorectal tissue in the HPA database.

(A): BRCA1, (B): CELF4, (C): HEXIM1, (D): NOVA1, (E): PNLDC1, (F): RRS1. 


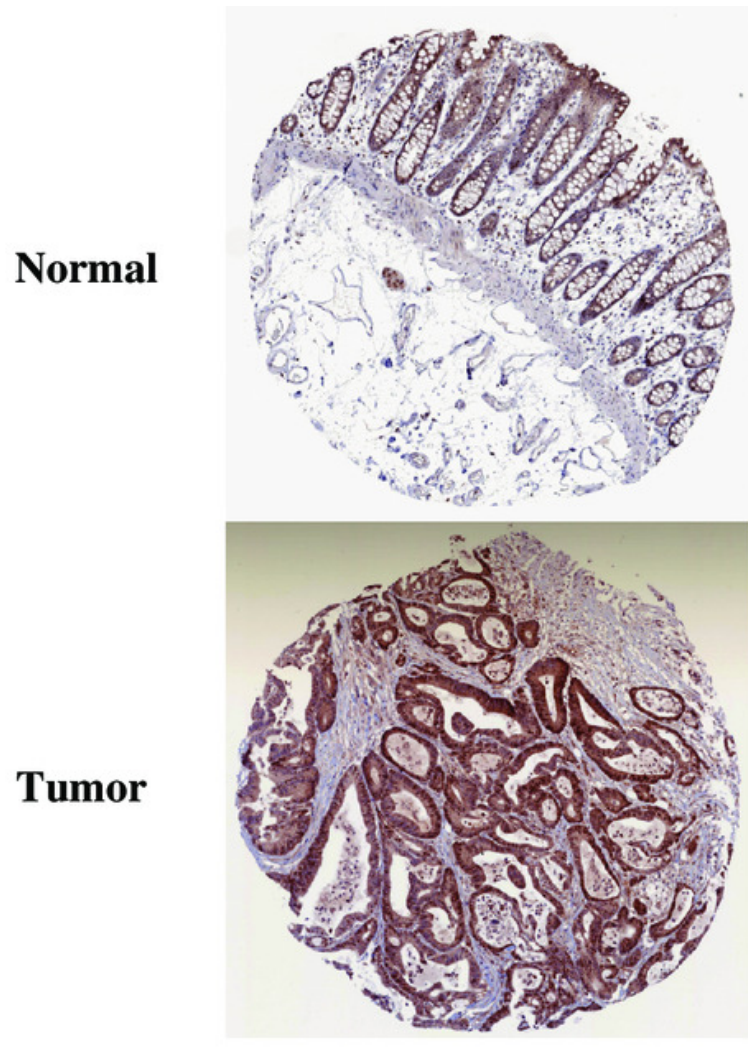

(A) BRCA1

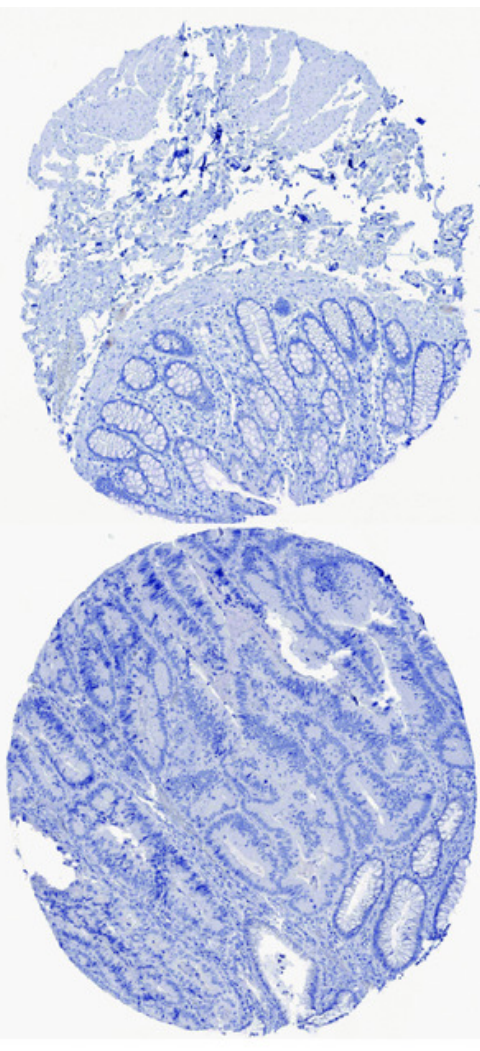

(B) CELF4

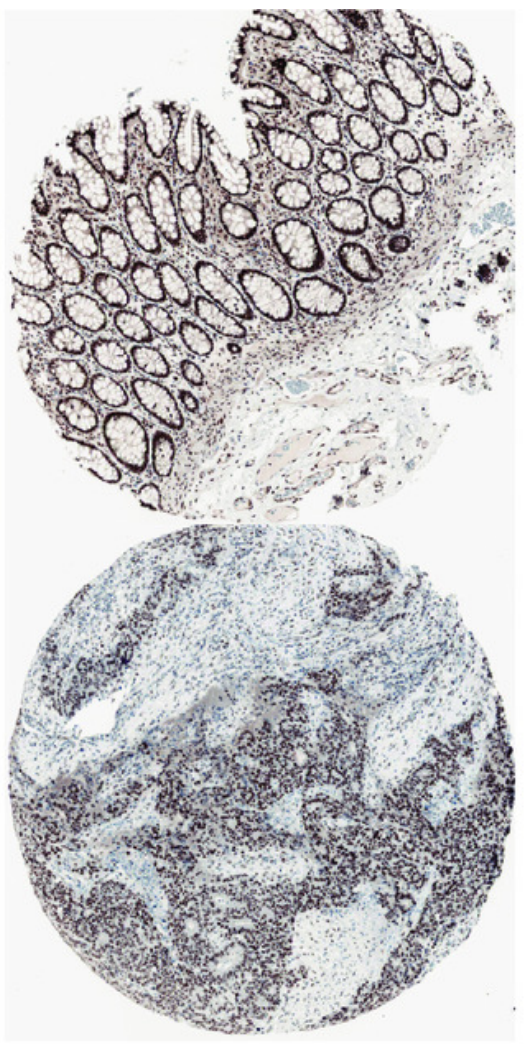

(C) HEXIM1

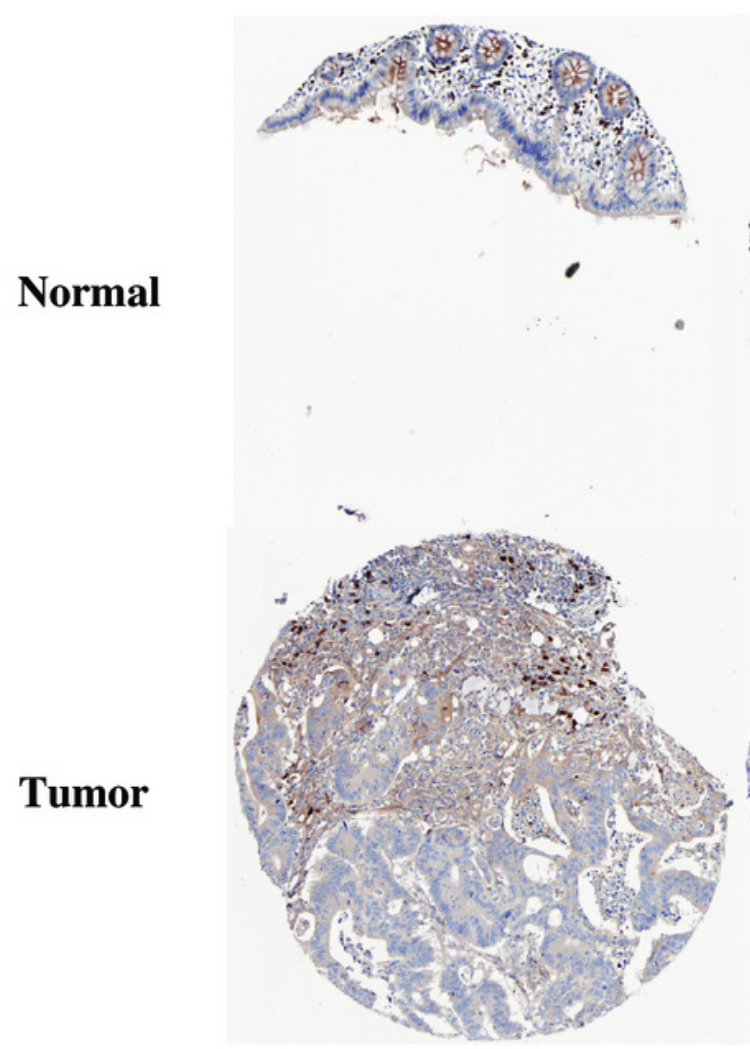

(D) NOVA1
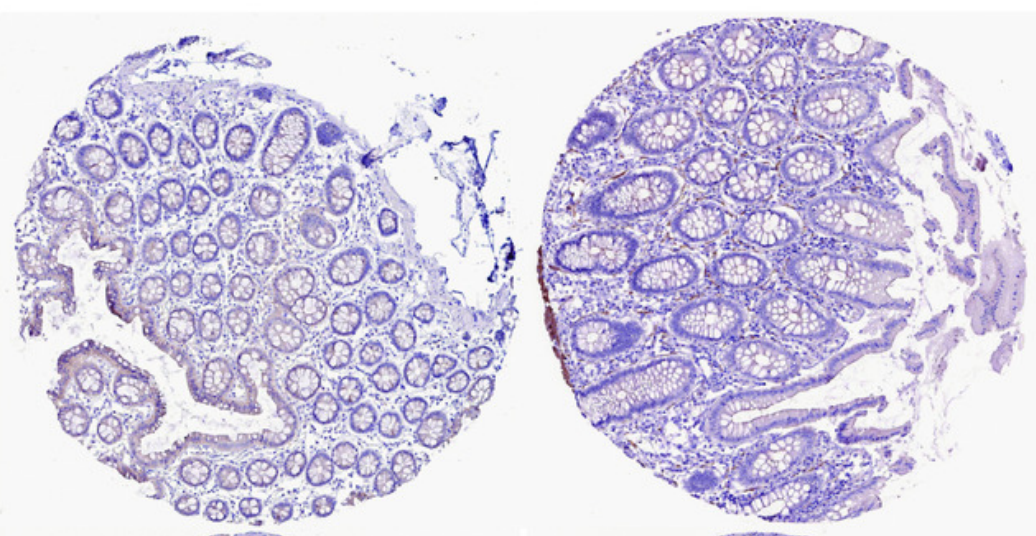

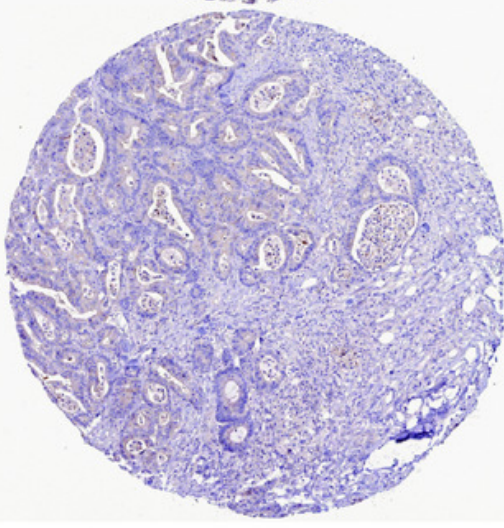

(E) PNLDC1

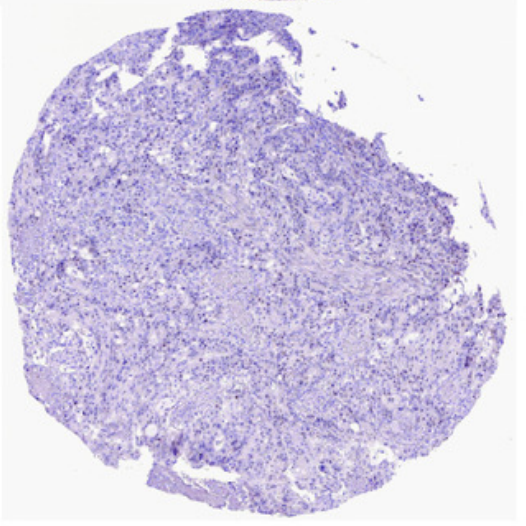

(F) RRS1 


\section{Table $\mathbf{1}$ (on next page)}

Table 1. Prognosis-related RBPs (multivariate Cox regression analysis) and prognosis model coefficient 
1 Table 1. Prognosis-related RBPs (multivariate Cox regression analysis) and prognosis model

\section{2 coefficient}

3

\begin{tabular}{|c|c|c|c|c|c|c|}
\hline RBPs & Official Full Name & Coefficient & HR & Lower $95 \%$ CI & Upper $95 \%$ CI & $P$-value \\
\hline PNLDC1 & PARN Like, Ribonuclease Domain Containing 1 & 0.2943 & 1.342146139 & 1.093946483 & 1.646658486 & 0.004792852 \\
\hline RRS1 & Ribosome Biogenesis Regulator 1 Homolog & 0.4835 & 1.621755576 & 1.170686306 & 2.246623313 & 0.003641482 \\
\hline HEXIM1 & HEXIM P-TEFb complex subunit 1 & 0.3609 & 1.434554531 & 1.045118889 & 1.969102963 & 0.025545301 \\
\hline PPARGC1A & PPARG Coactivator 1 Alpha & -0.1757 & 0.838828662 & 0.668974465 & 1.05180924 & 0.127906411 \\
\hline PPARGC1B & PPARG Coactivator 1 Beta & -0.3660 & 0.693496854 & 0.464111999 & 1.036253939 & 0.074071497 \\
\hline BRCA1 & BRCA1 DNA Repair Associated & -0.4157 & 0.659848664 & 0.428493546 & 1.016118593 & 0.059110143 \\
\hline CELF4 & CUGBP Elav-like Family Member 4 & 0.5460 & 1.72633526 & 1.307387604 & 2.279533186 & 0.000118191 \\
\hline AEN & Apoptosis Enhancing Nuclease & 0.4375 & 1.548761254 & 1.169130348 & 2.051662954 & 0.002295159 \\
\hline NOVA1 & NOVA Alternative Splicing Regulator 1 & 0.1639 & 1.178115785 & 0.957878215 & 1.448990884 & 0.120567185 \\
\hline
\end{tabular}

4 
Table 2 (on next page)

Table 2. The prognostic value of different clinical characters in the train group. 
1 Table 2. The prognostic value of different clinical characters in the train group.

\begin{tabular}{|c|c|c|c|c|c|c|}
\hline & \multicolumn{3}{|c|}{ Univariate prognostic analysis } & \multicolumn{3}{|c|}{ Multivariate prognostic analysis } \\
\hline & HR & $95 \% \mathrm{CI}$ & $P$-value & HR & $95 \% \mathrm{CI}$ & $P$-value \\
\hline Age & 1.0360 & $1.0158-1.0567$ & 4.49E-04 & 1.0529 & $1.0316-1.0747$ & 7.85E-07 \\
\hline Gender & 1.0782 & $0.7154-1.6251$ & 0.7189 & 0.8547 & $0.5624-1.2989$ & 0.4621 \\
\hline Stage & 2.4378 & $1.9233-3.0901$ & $1.75 \mathrm{E}-13$ & 1.7652 & $0.8994-3.4643$ & 0.0986 \\
\hline $\mathrm{T}$ & 2.9977 & $1.9876-4.5212$ & $1.64 \mathrm{E}-07$ & 1.6070 & $1.0023-2.5766$ & 0.0489 \\
\hline M & 4.8650 & $3.1979-7.4012$ & $1.47 \mathrm{E}-13$ & 1.5751 & $0.6184-4.0119$ & 0.3408 \\
\hline $\mathrm{N}$ & 2.0793 & $1.6380-2.6396$ & $1.81 \mathrm{E}-09$ & 1.1524 & $0.7623-1.7422$ & $7.54 \mathrm{E}-04$ \\
\hline Riskscore & 1.0843 & $1.0566-1.1127$ & 8.63E-10 & 1.0827 & $1.0496-1.1168$ & $5.08 \mathrm{E}-07$ \\
\hline
\end{tabular}

2 


\section{Table 3 (on next page)}

Table 3. The prognostic value of different clinical characters in the validation group. 
1 Table 3. The prognostic value of different clinical characters in the validation group.

\begin{tabular}{|c|c|c|c|c|c|c|}
\hline & \multicolumn{3}{|c|}{ Univariate prognostic analysis } & \multicolumn{3}{|c|}{ Multivariate prognostic analysis } \\
\hline & HR & $95 \% \mathrm{CI}$ & $P$-value & HR & $95 \% \mathrm{CI}$ & $P$-value \\
\hline Age & 1.0238 & $1.0115-1.0362$ & $1.32 \mathrm{E}-04$ & 1.0317 & $1.0191-1.0445$ & $6.45 \mathrm{E}-07$ \\
\hline Gender & 1.1140 & $0.8394-1.4785$ & 0.4547 & 1.2753 & $0.9587-1.6963$ & 0.0948 \\
\hline Stage & 1.9205 & $1.5559-2.3704$ & 1.23E-09 & 0.7029 & $0.4540-1.0884$ & 0.1140 \\
\hline $\mathrm{T}$ & 1.9591 & $1.4880-2.5794$ & $1.65 \mathrm{E}-06$ & 1.6453 & $1.2436-2.1767$ & 0.0005 \\
\hline M & 5.3710 & 3.7963- 7.5989 & $2.20 \mathrm{E}-21$ & 6.9200 & $3.6552-13.1009$ & $2.85 \mathrm{E}-09$ \\
\hline $\mathrm{N}$ & 1.4507 & $1.2115-1.7371$ & $5.20 \mathrm{E}-05$ & 1.5135 & $1.1301-2.0269$ & 0.0054 \\
\hline Riskscore & 1.2161 & $1.0965-1.3487$ & $2.12 \mathrm{E}-04$ & 1.1347 & $1.0056-1.2805$ & 0.0403 \\
\hline
\end{tabular}

2 